\title{
Das Blutbild der Tuberkulose im Hochgebirge.
}

\author{
Von \\ Dr. W. Knoll. \\ (Aus der Bündner Heilstätte Arosa. [Chefarzt: Dr. W. Knoll].) \\ (Eingegangen am 25. 11. 1921.)
}

In den folgenden Mitteilungen soll nacheinander für das Gebiet der Erforschung des Blutes sowohl nach der morphologischen Seite wie nach den in jüngerer und jüngster Zeit ausgearbeiteten Methoden der Viskosimetrie des Serums, der Refraktionsbestimmung desselben, der Spektroskopie, sowic anderer im Verlaufe der Untersuchungen sich ergebender Verfahren, an Hand längerer Untersuchungsreihen, die die Ernittlumg eines Mittelwertes möglich machen, alles das zusammengetragen werden, was für die Veränderungen, die das gesamte Blutbild bei Tuberkulose während der Hochgebirgskur erleidet, charakteristisch ist. Der Stoff ist so gegliedert, da $\beta$ erst die eigenen Untersuchungen besprochen werden, hernach die Literatur zu dem vorliegenden Kapitel und deren kritische Würdigung mit Rücksicht auf die eigenen Untersuchungsreihen.

Wir glauben damit ein Gebiet im Zusammenhang besprechen zu können, dessen einzelne Komponenten wohl schon wiederholt durchgearbeitet wurden, für die aber eine zusammenfassende Darstellung bis jetzt nicht vorhanden ist.

Die Verhältnisse in Arosa sind dazu besonders günstig, weil hier ein ziemlich großes Material beisammen ist und auch die Höhe von $1800 \mathrm{~m}$ gegenüber dem Tiefland eine klimatisch genügende Differenz ergibt, um eventuelle dadurch bedingte Unterschiede herauszufinden.

Wir glauben darum, durch die vorliegende Form eine Lücke auszufüllen und denjenigen, die sich mit der Heilung der Tuberkulose im Hochgebirge befassen, diesen und jenen Hinweis auf die speziellen Verhältnisse, unter denen unsere Kranken leben, sowie auch für die Prognosestellung geben zu können.

\section{Mitteilung.}

\section{Morphologie. ${ }^{1}$ )}

$$
\text { Von }
$$

$$
\text { Dr. W. Knoll, und Dr. H. Graf, }
$$

Die Gründe, die uns zur Inangriffnahme der vorliegenden Untersuchungen veranlaßten, liegen kurz in folgendem. Über die physiologischen Veränderungen der Morphologie des Blutes beim Übergang von tieferen in höhere Gegenden liegen seit den grundlegenden Untersuchungsreihen der Miescherschen

1) Teilweise mitgeteilt in der wissenschaftlichen Sitzung der Schweizer. Vereinigung der Tuberkuloseärzte 1921 in Zürich und in der Sitzung des Ärzte-Vereins Arosa vom 2. September 1921. 
Schule zahlreiche, teils bestätigende, teils aber auch abweichende Resultate vor, von denen wir besonders die Arbeiten Bürkers und seiner Nachfolger nennen möchten. Diese Untersuchungen haben uns eines sicher gebracht, den Beweis der tatsächlichen Vermehrung der $\mathrm{R}$ und des Hgb beim Úbergang von Orten höheren Luftdruckes zu solchen niedrigeren Druckes. Die Ursache wird heute wohl allgemein in dem verminderten Sauerstoffpartiardruck in größerer als der gewohnten Höhe angenommen.

Bis dahin gehen die Meinungen der großen Mehrzahl parallel. Aber schon die quantitativen Verhältnisse bringen erhebliche Differenzen. Während in den älteren Arbeiten [Egger, Suter, Karcher Veillon (1), auch Rö misch (1)] die Vermehrung eine ganz erhebliche ist und Zahlen von 6-7 Mill. die Regel, geringere aber die Ausnahme bilden, hat die Bürkersche (1) erste Untersuchung nur Werte von 5,5 Mill. gebracht und Wanner (1) sowie Craandyk (2) kommen zu ähnlichen Zahlen.

Man hat versucht, eine Proportion herzustellen zwischen der Höhe über Meer und der normal dort vorhandenen Zahl der $\mathrm{R}$ im Kubikmillimeter Blut. Bezügliche Zahlen gibt Nägeli ( 1 ) in seinem Lehrbuch wieder, macht aber bereits Fragezeichen zu einigen von ihnen. Wenn es auch sehr schön wäre, falls ein solches absolutes Gesetz bestünde, weil es uns eine viel sicherere therapeutische Wegleitung an die Hand gäbe, so vernachlässigen diese Übcrlegungen doch eines, das ist die individuelle Schwankung, die doch einen viel zu großen Raum einnimmt, als daß sie sich auf ein solches meteorologisches Schema cinstellen ließe.

Diese individuellen Schwankungen sind es denn auch, die nach unserer Auffassung die meisten Differenzen auf dem Gewissen haben und denen gegenüber die Technik nicht in dem Maße ins Gewicht fällt, wie es verschiedentlich behauptet worden ist. Die Untersuchungsmethoden, die uns heute zur Zählung der $\mathbf{R}$ und $W$ im Blute zur Verfügung stehen, sind bei gewissenhafter Handhabung in technisch geschulter Hand alle brauchbar und geben keine so wesentlichen Differenzen, daß dadurch die Unterschiede erklärbar wären. Daß tatsächlich sowohl die Mieschersche Schuleals nach ihr die Bürkersche mit minutiöser Exakthcit gearbeitet haben, steht für uns über allem Zweifel. Wir dürfen nach unseren eigenen Erfahrungen auch sagen, daß Vergleichsuntersuchungen mit verschiedenen Instrumentarien unserer eigenen Apparatur nach Th o ma mit Th o m a und Breuerkam mern und zugehörigen Mischpipetten im Vergleiche zu den uns cbenfalls zur Verfügung stehenden Pipetten und Kammern von Römisch, mit denen er seine Arbeiten seinerzeit ausgeführt hat, und endlich das auf anderen Prinzipien aufgebaute Arbeiten mit dem Sahli-Hayemschen Instrumentarium grundsätzlich bei denselben Menschen auch dieselben Resultate gab, wobei der Fehler jedenfalls nicht über dem mittleren Fehler lag. Genauere Zahlen folgen später. Zudem haben wir an einer großen Reihe im Durchschnitt die Zahlen von Bürker, wie er sie als Durschchnitt seiner Serien bekommt, ebenfalls erhalten, so daß damit auch die Übereinstimmung mit den Resultaten der Bürkerkammer erwiesen ist. Also auf die Apparatur können die abweichenden Resultate nicht bezogen werden. Ebensowenig kann die Person eines mit seinem Instrumentarium eingeübten Untersuchers in Frage kommen. Es bleibt als letztes das Objekt, vielmehr die Objekte, eine Auffassung, die sich uns im 
Verlaufe der zahlreichen Untersuchungen und Kontrollen aufdrängen mußte und die es ganz zwanglos erklärt, warum verschiedene, gewissenhafte Untersuchér zu abweichenden Resultaten selbst am selben Orte gelangen mußten. Die geringe Zahl der untersuchten Personen, die den einzelnen Untersuchern zur Verfügung standen, hatte neben dem Vorteil der mehrfachen Untersuchungen und der Beobachtung derselben Versuchsperson während längerer Zeit doch den großen Nachteil eines rein individuellen, nicht weiter kontrollierbaren Resultates, dessen Verallgemeinerung doch nicht ohne weiteres anging.

Wir wollten darum unsere Untersuchungen selbst auf Kosten der dann nicht in dem von anderen geübten Maße möglichen Wiederholung am selben Objekt gerade auf eine möglichst große Zahl untersuchter Personen ausdehnen, um die individuellen Schwankungen durch Errechnung einer Mittelzahl so gut als möglich auszuschalten.

Es mag auffallen, daß wir physiologische Tatsachen als welche die Blutuntersuchungen im Hochgebirge bisher stets aufgefaßt und gehandhabt worden sind, auf pathologisches Gebiet einfach übertragen. Wenn auch die Bedingungen, unter denen die Anpassung vom kranken Menschen geleistet werden $\mathrm{muB}$, in Einzelheiten abweichen mag, grundsätzlich muß doch die Reaktion denselben Weg machen, ist es doch immer und unter allen Versuchsbedingungen letzten Endes die Anpassung der Knochenmarksfunktion an die veränderten Lebensbedingungen. Wir befinden uns also auf durchaus wissenschaftlichem Boden und haben zudem in Römisch (1), Kündig (3), van Vornfeld (4), Schultz (5), Craandyk (2) bereits Vorgänger. Unsere Untersuchungen beschäftigen sich also in erster Linie mit den von den physiologischen, für den Ort ebenfalls mit bestmöglicher Genauigkeit festgestellten Verhältnissen abweichenden pathologisch-physiologischen Befunden, wie wir sie bei unseren an irgendeiner Tuberkulose leidenden Kranken gefunden haben. Wie überhaupt kann es sich naturgemäß nicht um Blutkrankheiten handeln, sondern die Verhältnisse im strömenden Blut geben uns nur einen, wenn auch bescheidenen Anhaltspunkt darüber, wie sich das Knochenmark als funktionierendes Organ zu den veränderten Bedingungen stellt, in die es durch Verbringung ins Hochgebirge aus einem wesentlich tiefer gelegenen Orte, an den es seine Funktion angepaßt hatte, versetzt wird. Vielleicht ergeben sich im Verlaufe der Untersuchungen auch einige Streiflichter auf die heute hochaktuelle Frage nach der kolloid-chemischen Natur der Vorgänge innerhalb des strömenden Blutes, wie sie Zangger (6) als erster ausgesprochen hat und wie sie vor kurzem von Sahli (7) in überzeugender Weise dargelegt wurden.

In erster Linie schien es uns wichtig, ein für unseren Ort geltendes Mittel bei gesunden Einwohnern, Männern und Frauen, also ein lokales physiologisches Mittel festzustellen. Hatten wir einmal ein solches Mittel, so mußten wir versuchen, nachzuweisen, ob sich bei unseren Kranken an möglichst großer Anzahl dieselben Verhältnisse ergeben würden, wenn nicht welche Abweichungen immer im Mittel von der Norm nachweisbar waren und dann, welche Zeit es brauchte, um die Einstellung auf das indfviduelle Optimum, als welches wir die bleibende Vermehrung ansehen müssen, zu erreichen. Dasselbe wurde dann wie für das rote so auch für das weiße Blutbild durchgeführt. Es ergaben sich so mehrere, untereinander vergleichbare Reihen, die uns gestatten sollten, 
einen tieferen Blick in die im strömenden Blut nachweisbaren Anpassungserscheinungen zu tun, einmal gleich nach dem Utbergang von tiefer gelegenen Orten auf die Höhe von $1800 \mathrm{~m}$ und dann auch über den ganzen Ablauf dieses Vorganges bis zum Eintritt der bleibenden Veränderungen.'

Dabei war mit folgenden Fehlerquellen von vornhercin zu rechnen: Zeitpunkt der Blutentnahme, Wahl des Ortes, Ausschaltung eventuell kleiner technischer Ungenauigkeiten, die das Resultat unvergleichbar machen konnten.

Es wurden folgende Untersuchungsreihen durchgeführt: 1. Gesunde Männer und Frauen. 2. Tuberkulosen aller Formen und Stadien, zweimal untersucht, und zwar innerhalb der ersten 24 Stunden und am Schlusse der Kur. 3 Tuberkulosen aller Formen und Stadien, 3-4 mal und mehr untersucht innerhalb der ersten 24 Stunden nach 8 Tagen, nach 14 Tagen und am Schlusse der Kur. Im ganzen wurden untersucht: 20 gesunde Männer, 20 gesunde Frauen, 200 Patienten der ersten Serie, 150 Patienten der zweiten Serie.

Die vergleichenden Untersuchungen mit verschiedenen Methoden wurden an 22 gesunden Männern und Frauen vorgenommen.

Wir besprechen nacheinander: 1. Die Technik, 2. das rote Blutbild, 3. das weiße Blutbild, 4. die Literatur. Zusammenfassung am Schlusse.

Den Untersuchungen an Kranken gehen jeweils die entsprechenden Befunde an Gesunden voraus ${ }^{1}$ ).

\section{A. Technik.}

Es ist klar, daß eine genaue Technik wesentlich zum richtigen Resultat morphologischer Blutuntersuchungen beitragen muß, die Bedeutung jedoch, die der Methodik bzw. Kammern und Pipetten, namentlich von späteren Untersuchern für die Resultate zugeschrieben wurde, besitzt sie nach unseren Erfahrungen mit verschiedenen Kammern, Pipetten und zwei differenten Methoden jedoch nicht. Näheres siehe später.

Wir entnahmen das Blut zwischen 9 und 11 Uhr. Die erste Entnahme geschah an dem der Aufnahme folgenden Tage im Bett, die anderen je nach dem Zustande des Kranken im Bett oder ambulant nach mehrstündiger Ruhe. Entnahmestelle die Fingerkuppe, nachdem der Finger für mehrere Minuten in warmes Wasser gehalten worden war. Benutzung vom zweiten Tropfen an, und zwar wurde die Untersuchung stets in derselben Reihenfolge angestellt: 1. Hämoglobinbestimmung nach der Sahlischen Skala, die angegebenen Zahlen entsprechen also den Graden des Sahlischen Hämoglobinometers, sind demnach keine Prozentzahlen.

2. Zählung der $\mathrm{R}$, genaues Ansaugen bis zur Marke war Vorbedingung; falls ungenau, mußte eine neue Pipette beschickt werden.

Die Pipetten wurden nach Gebrauch sofort mit Alc. abs. und hernach mit Benzin ausgeblasen resp. trocken gesogen, was sich uns gut bewährte. Es ist wichtig, daB die Pipetten völlig trocken sind, da selbst kleinste Flüssigkeitströpfchen ungenaue Resultate ergeben.

1) Mit Rücksicht auf die Druckkosten haben wir auf die ausführlichen Tabellen verzichtet und geben nur eine solche der Gesamtresultate bezüglich R Hgb und F J am Schlusse des ersten Abschnittes. 
Nach dem Ansaugen der Hayemschen Flüssigkeit ist es wichtig, sofort das Schütteln anzuschließen, weil sonst ein Verbacken der $\mathbf{R}$ statthaben kann, das nie mehr ganz verschwindet und die Resultate stark und bis zur Unbrauchbarkeit beeinflußt. Ebenso wichtig ist es, die Kammer unmittelbar nach Aufhören des Schüttelns, d. h. bei uns nach 5 Minuten zu erstellen. Sie wurden so hergestellt, daß zunächst das Deckglas soweit über die Kammer geschoben wurde, bis es nur noch einen wenige Millimeter breiten Saum derselben offen ließ. Beim Zufließenlassen der Mischung aus der Pipette breitet sich diese sofort und gleichmäßig in der Kammer aus und ein leichter allseitiger Druck außerhalb der Kammer auf das Deckglas genügt, um die bekannten Newtonschen Ringe, ohne die eine Kammer unbrauchbar ist, erscheinen zu lassen.

Wir verwendeten sowohl Thoma- wie Breuerkammern mit demselben Resultat. Die Kammern wurden jeweils miteinander verglichen, wobei sich Fehler ergaben, die innerhalb der Fehlergrenze lagen.

Um eventuell Abweichungen zwischen unserem Instrumentarium und demjenigen anderer Autoren festzustellen, arbeiteten wir einmal mit dem Instrumentarium von Römisch (1), das uns von ihm in bereitwilligster Weise längere Zeit überlassen wurde. Die Resultate stimmten durchaus überein. Ferner haben wir, um Abweichungen in der Auszählung, die uns hätten Schwierigkeiten bereiten können, auszuschalten, eine bestimmte Anzahl von Patienten sowohl mit unserer bewährten Methode als auch nach dem Verfahren von SahliHayem untersucht. Diese Reihe von 22 gesunden Einwohnern von Arosa ergab eine mittlere Abweichung nach beiden Seiten von $3 \%$, also eine mittlere Gesamtabweichung von $6 \%$. Die maximale Differenz wurde einmal zu $6,82 \%$; 2 mal zu 3\% festgestellt, alle anderen Werte wichen in geringerem Grade ab. Die Abweichungen betrafen in positivem Sinne je 11 mal die Methode von Sahli-Haye $\mathrm{m}$ und ebenso $11 \mathrm{mal}$ diejenige nach Thoma-Zeiß.

Es war somit nachgewiesen, daß mit beiden Methoden dieselben Resultate erzielt wurden. Die Sahlische Methode ist naturgemäß die sicherere, wenn man weniger Übung hat, weil sie mit größeren Zahlen arbeitet und die Vergrößerung für genaue Zählungen sehr angenehm ist. Wir haben dann auch unsere gewöhnlichen Zählungen mit dem Okular 3 und Objelztiv 6 Leitz oder DD Zeiß vorgenommen und uns dabei von der Sicherheit, mit der man zählt, überzeugen können.

In der Thoma- oder Breuerkammer wurden jeweils 5 mal 20 kleine Quadrate $=100$ kleine Quadrate gezählt. Ergaben sich Differenzen von mehr als $30 \mathrm{R}$ pro 20 Quadraten, so wurde dieselbe Anzahl, also nochmals 100 durchgezählt. Kammern mit mehrfachen Differenzen über $50 \mathrm{R}$ betrachteten wir als unbrauchbar und wiederholten den Versuch. Eventuelle Größenunterschiede der $\mathrm{R}$ fallen bei diesen Vergrößerungen viel besser auf als bei den kleinen, auch können einem so keine Mikrozyten und kleine Poikilozyten entgehen. Die pathologischen Befunde an den $\mathbf{R}$ wurden jeweils schon in der Kammer festgestellt und nachher im gefärbten Präparat nachkontrolliert.

2. Weiße Blutkörperchen W. Es wurden stets alle 9 Rechtecke der Brenerkammer, die auf die oben beschriebene Weise beschickt. war, durchgezählt. Zur prozentualen Auszählung wurden die Ausstriche in der Weise hergestellt, daß der Tropfen mit der Kante eines schräg gestellten geschliffenen Objektträgers auf einem zweitén Objektträger ausgezogen wurde, wodurch jede Quet- 
schung unmöglich wird. Der Tropfen muß ganz frisch sein, weil sich sonst die $W$ nur am Rande des Ausstriches befinden, wodurch dieser unbrauchbar wird. Er wird zweckmäßig im Hängen abgenommen.

Es wurde jeweils ein dünner und ein dickerer Ausstrich gemacht, um besondere Verhältnisse nachkontrollieren zu können.

Die Präparate wurden dann 24 Stunden an der Luft getrocknet, hernach 3-5 Minuten mit absolutem Methylalkohol fixiert und nach Giemsa gefärbt. So vorbehandelt wird die Färbung konstant und sehr gut und hat uns nie im Stich gelassen. Kürzeres Lufttrocknen dagegen läßt oftmals bei der Färbung der n. Granulation zu wünschen übrig.

Insbesondere war es uns dabei stets möglich, mit absoluter Sicherheit Monozyten, Lymphozyten und Plasmazellen auseinander zu halten.

Die von Liebmann (8) angegebene Feuchtfixation, die uns der Autor vermittelte, hat uns nach eingehenden Versuchen nicht befriedigt. Die Veränderungen, die namentlich die Kerne dabei erleiden, sind doch noch erheblichere als beim vorhergehenden Lufttrocknen, da dabei der Wasserentzug ein zu plötzlicher ist. Kn oll (9) hat auf diese Verhältnisse in anderem Zusammenhang bereits vor mehreren Jahren hingewiesen und auch experimentell die Veränderungen der äußeren Form des Leukozytenkerns am überlebenden Objekt unter dem Einfluß eines zugefügten Fixationsmittels studiert. Auch damals hat sich absoluter Alkohol sowie Methylalkohol als das noch am wenigsten verändernde Mittel erwiesen.

Die Feststellung der Azurgranulation der Ly, wie sie in neuester Zeit von Schenk (10) versucht wurde, haben auch wir an mehr als 40 Fällen nachgeprüft mit dem Resultat, daß diese Körnchen beim selben Patienten von 2 bis $80 \%$ im Vorkommen schwanken können, so daß damit für unsere Zwecke nichts anzufangen war.

Die Beurteilung des weißen Blutbildes nach Arneth (11) haben wir ebenfalls nicht herangezogen einmal wegen des seinerzeit von $\mathrm{Brugsch}$ und Schilling (12) auf Grund ihrer Dunkelfelduntersuchungen an überlebenden Leukozyten gegen die Theorie erhobenen prinzipiellen Bedenken, indem diese Autoren nachweisen konnten, daß die Anzahl der Kernsegmente eines und desselben Exemplares während der Beobachtungszeit an Zahl schwanken konnte und weil auch die Beobachtungen von Knoll (9) am selben Objekt zu dem gleichen Resultat gelangt waren. Es entscheidet darum unseres Erachtens nicht bloß die Kernform über das Alter oder die relative Jugend eines Leukozyten, hier vom neutrophilen Typus, sondern die ganze Kernstruktur, wobei erst noch die Frage unentschieden bleibt, ob der eine oder andere während des Lebens Gelegenheit zu größeren Formveränderungen hatte, während sie anderen gleichalten fehlte. Der Augenblick der Fixation mit seinen bereits oben erwähnten nachher nicht mehr kontrollierbaren Einflüssen auf die Kerngestalt spricht hier auch ein Wort mit, das bisher unseres Erachtens in der Behandlung dieser Frage zu wenig berücksichtigt worden ist.

\section{B. Das rote Blutbild.}

Vorgängig der Zasammenstellung unserer Untersuchungen an Kranken sei das Resultat der Blutuntersuchungen an 40 klinisch gesunden und voll 
arbeitsfähigen Erwachsenen Einwohnern von Arosa mitgeteilt, durch das wir uns ein Bild des mittleren Status machen wollten, wie er auf unserer Höhe von $1800 \mathrm{~m}$ besteht. Wir waren überrascht, zu finden, daß die Zahlen auch nicht annähernd so hoch waren wie die früher vom selben Orte von Egger (1) und Römisch (1) mitgeteilten. Diese Abweichungen veranlaßten uns dann zu den bereits mitgeteilten Vergleichsuntersuchungen mit dem Verfahren von Haye m-Sahli.

\begin{tabular}{|c|c|c|c|c|c|}
\hline Mitt & $\mathrm{R}$ & $\mathrm{Hgb}$ & $\mathbf{F I}$ & $\begin{array}{c}\text { Hgb Quot } \\
\text { nach Cra andyk }\end{array}$ & Ges \\
\hline $\begin{array}{l}\text { bei Männern } \\
\text { bei Frauen } . \\
\text { b. }\end{array}$ & $\begin{array}{l}\text { 5,42 Mill. } \\
5,23 \text {, }\end{array}$ & $\begin{array}{l}95,9 \\
87,3\end{array}$ & $\begin{array}{l}0,87 \\
0,83\end{array}$ & $\begin{array}{l}30,7 \mathrm{~g} \times 10^{-12} \\
28,9 \mathrm{~g} \times 10^{-12}\end{array}$ & \\
\hline
\end{tabular}

Es befinden sich darunter 5 Männer und 2 Frauen, deren R-Bestand pro Kubikmillimeter nicht auf der Höhe des für das Tiefland normalen steht.

Wir haben diese Leute mehrfach untersucht und unsere ersten Resultate bestätigt gefunden. Bei diesen Leuten steht der abnorm niedrigen R-Zahl eine abnorm hohe Hgb-Zahl gegenüber; der F I ist also erhöht.

Die Mittelzahlen sind folgende:

$\begin{array}{llccc} & & \mathbf{R} & \mathrm{Hgb} & \mathrm{F} \mathrm{J} \\ \mathbf{5} \text { Männer . } & \mathbf{4 , 3 2} & \mathbf{9 3} & 1,07 \\ 2 \text { Frauen . } & \mathbf{4}, 35 & 85 & \mathbf{0 , 9 7}\end{array}$

Wir erklären uns die Sache so, daß bei einem gewissen, mit unserem Material nicht prozentual zu berechnenden Teil der Untersuchten die Kompensation im Hochgebirge nicht durch eine Vermehrung der $\mathbf{R}$, sondern eine Vermehrung und, wie wir annehmen müssen, auch bessere Ausnützungsmöglichkeit des Hgb. Gehaltes des einzelnen $R$ erreicht wird. Das Wesentliche ist doch die optimale Ausnützung des vorhandenen Hgb-Gehaltes. Ob dies auf dem Wege der absoluten R-Vermehrung oder auf dem der bloßen Erhöhung des Hgb-Gehaltes oder wie meist auf dem Wege einer Kombination beider Komponenten erfolgt, ist unwesentlich, wesentlich allein ist die Ausnützungsmöglichkeit des Hgb und diese ist wohl durch beide Extreme gegeben. Wir stehen hier unseres Erachtens vor individuell wechselnden Reaktionsformen, die noch nicht genügend abgeklärt sind, deren Erwähnung uns aber doch wichtig erscheint zum Verständnis der an und für sich schon komplizierten Vorgänge.

Wir werden später wieder auf solche Fälle zurückkommen und erwähnen nur noch, daß es sich bei den genannten Gesunden um junge Männer und Frauen handelte, die keinerlei klinische Krankheitszeichen boten und auch zum Teil große Anstrengungen, wie es die Skiwettfahrten im Hochgebirge sind, ohne Störungen ihrer Gesundheit absolvierten. Vier von ihnen kennen. wir ärztlich seit 5 Jahren und haben sie wiederholt vor und nach solchen An. strengungen untersucht.

Auch die weiblichen Gesunden kennen wir seit Jahren.

Die Erklärung für dieses Vorkommnis setzt verschiedene Begriffe voraus, die wir hier in Kürze zusammenstellen möchten.

Stellt man sich den Vorgang des Gasaustausches zwischen der Alveolarluft und dem $\mathrm{Hgb}$ des $\mathrm{R}$ als einen Vorgang vor, der mit der Oberflächenspannung im kolloid-chemischen 'Sinne eng zusammenhängt, so muß man den Ort, wo sich dieser Vorgang abspielt, an die Oberfläche der $R$ verlegen. 
Eine hohe R-Zahl wird also diese Oberfläche vergrößern, und zwar können wir diese Oberfläche pro Kubikmillimeter berechnen. Herr Ingenieur Scheller hatte die Freundlichkeit, dies für uns zu tun. Es würde zu weit führen, hier die mathematische Ableitung in extenso wiederzugeben, es genügt, daß für Annäherungswerte, wie sie ja auch unsere Blutkörperchenzählungen darstellen, die Formel gilt:

$$
F=2 D^{2}
$$

wobei $\mathbf{F}=$ Oberfläche eines $\mathrm{R}, \mathrm{D}=$ Durchmesser eines $\mathrm{R}$ zu setzen ist. Der mittlere Durchmesser eines normalen $R$ beträgt nach $N a ̈ g e l i ~(1) ~ 7,5 \mu$, so daß die Oberfläche $=112,5 \mu^{2}$ wäre. Die Gesamtoberfläche aller $R$ im Kubikmillimeter, die für uns in Betracht kommt, betrüge somit 562,5 Mill. $\mu^{2}$. für Männer, 546,25 Mill. $\mu^{2}$ für Frauen. Unter normalen Bedingungen stehen für diese Oberfläche beim Mann $13,84 \mathrm{~g} \cdot 10^{-5} \mathrm{Hgb}$ beim Mann, 12,11 g . 10-5 $\mathrm{Hgb}$ bei Frauen zur Verfügung.

Wenden wir diese Rechnung auf unsere Zahlen an, so bekommen wir im Mittel folgende Werte nach nachstehendem Beispiel:

$\mathrm{R}=$ sei 5,0 Mill., $\mathrm{Hgb}=80$ Sahli, dann ist $\mathrm{F}=562,5$ Mill. $\mu^{2}$, das HgbGewicht pro $100 \mathrm{ccm}$ nach der Formel $\frac{80}{100} \cdot 1,73=13,84$ pro $\mu^{2}$,also $13,84 \cdot 10^{-5} \mathrm{~g}$.

Der Oberflächenquotient bestimmt sich dann nach der Gleichung

$$
\begin{gathered}
\frac{F}{\text { Hgb pro } \mu^{2}}=\text { Oberflächenquotient. } \\
\mathrm{F}=562,5 \mu^{2} \cdot 10^{8} \quad \text { Hgb }=13,84 \cdot 10^{-5}
\end{gathered}
$$

Dies ergibt als Oberflächenquotient $464,2 \mu^{2}$, d. h. für die Hgb-Einheit steht eine Oberfläche von $464,2 \mu^{2}$ zur Verfügung.

Für unsere Zahlen ergeben sich folgende Faktoren:

I. Normale Werte im Tiefland:

$$
\begin{array}{lccccc} 
& \mathrm{R} & \mathrm{F} & \mathrm{Hgb} & \text { pro } \mathrm{mm}^{3} & \mathrm{O} \mathrm{Q} \\
\mathrm{M} & \mathbf{5 , 0} & \mathbf{6 6 2 , 5} & 80 & 13,84 & \mathbf{4 6 4 , 2} \mu^{2} \\
\mathrm{~W} & \mathbf{4 , 5} & \mathbf{5 4 6 , 2 5} & 70 & 12,11 & \mathbf{4 5 1 , 0} \mu^{2}
\end{array}
$$

2. Mittelwerte für unsere Gesunden im Hochgebirge:

$\begin{array}{lllllll}\text { M } & 5,42 & 609,75 & 95,9 & 16,59 & 367,4 & \mu^{2} \\ \text { W } & 5,23 & 588,68 & 87,3 & 15,10 & 369,2 & \mu^{2}\end{array}$

3. Mittelwerte der Gesunden vom Typus II:

$\begin{array}{lllllll}\text { M } & 4,32 & 486,0 & 93,0 & 16,09 & 320,6 & \mu^{2} \\ W & 4,35 & 489,47 & 85,0 & 14,70 & 332,9 & \mu^{2}\end{array}$

Es ergibt sich also sowohl bei dem Durchschnitt aller Gesunden als ganz besonders bei den Leuten vom Typus II ein beträchtliches Absinken der für die Hgb-Einheit zur Verfügung stehenden Oberfläche.

Das Verhältnis dieser beiden Größen gibt uns also ein angenähertes Bild der Beziehungen zwischen der Oberfläche und dem $\mathrm{Hgb}$ in dem Sinne, daß wir daraus ablesen können, ob der Gehalt an $\mathrm{Hgb}$ größer oder geringer ist als in der Norm, ob also nach unserer oben entwickelten Anschauung die Oberfläche des einzelnen $\mathrm{R}$ mehr $\mathrm{Hgb}$ speichert als normal oder umgekehrt. Wir kennen aus der Pathologie zwei Krankheitszustände, die in extremem Maße diese Ver- 
hältnisse bereits zum Ausdruck bringen, die Chlorose und die perniziöse Anämie. Die Chlorose zeigt große Oberfläche bei geringem Hgb-Gehalt der einzelnen $\mathrm{R}$, die perniziöse Anämie dagegen geringe Oberfläche bei starkem Hgb-Gehalt des einzelnen R. Die Kompensation wird wie hier bei den Gesunden, aber nicht mit vollem Effekt im ersten Falle durch bessere Ausnützung des vorhandenen, nicht weiter regenerationsfähigen $\mathrm{Hgb}$, im zweiten durch Vermehrung des Hgb im einzelnen $\mathrm{R}$ nach unserer Anschauung also durch eine Anreicherung des Hgb an der Oberfläche der $R$ erreicht.

Es gäbe also schon normalerweise zweierlei Reaktionsformen, die sog. „,normale", die in weitaus dem größten Prozentsatz aller Fälle statthat, die primäre Vermehrung der $\mathrm{R}$ und damit die Vergrößerung der Oberfläche mit besserer Ausnützungsmöglichkeit des $\mathrm{Hgb}$ und die eben geschilderte, die dasselbe auf anderem Wege durch Speicherung des im Überschuß gebildeten $\mathbf{H g b}$ an der Oberfläche der $\mathbf{R}$ erreicht. Eine Vermehrung der Rum unseren Durchschnitt, beispielsweise des I. Stadiums, also 0,72 Mill., kommt einer Oberflächenvermehrung um 81 Mill. $\mu^{2}$ gleich, also etwa $15 \%$ der Gesamtoberfläche. Die Oberfläche beträgt für den Durchschnitt unserer Gesunden bei Männern 619,75 Mill. $\mu^{2}$, für Frauen 588,68 Mill. $\mu^{2}$. Die Gesunden waren entweder in Arosa geboren oder als gesunde Arbeiter und Angestellte heraufgekommen und seither durch mindestens 2 Jahre in Arosa geblieben. Wir durften darum als Regel annehmen, daß in dieser Zeit die Anpassung an die veränderten Verhältnisse erfolgt sei und die gefundenen Zahlen der bleibenden Reaktion entsprechen. Immerhin konnten wir in einem Einzelfalle ohne nachweisbare Erkrankung feststellen, daß sich noch nach 3 Jahren sowohl die Zahl der R als die Hgb-Zahl hob. Immerhin dürfte es sich hier um eine enorm verzögerte Anpassung handeIn, die nicht als Norm gelten kann. Sie zeigt uns nur, in wie großen zeitlichen Grenzen die Anpassung individuell schwanken kann, so daß wir von vornherein darauf gefaßt sein müssen, die Reaktion nicht stets im gleichen Zeitraum erfolgen zu sehen und auch bei der quantitativen und qualitativen Seite der Frage sich dieser Faktor störend werde geltend machen. Jedes Individuum reagiert eben als solches, im ganzen genommen wohl in gleichem Sinne, im einzelnen dagegen außerordentlich wechselnd und zum voraus nicht berechenbar. Es ergeben sich daraus notgedrungen Differenzen zwischen den Resultaten der einzelnen Autoren, die mit diesen individuellen Schwankungen eng zusammenhängen und um so bemerkbarer sein müssen, je geringer die untersuchte Individuenzahl ist. Es war uns darum zu tun, durch eine möglichst große Reihe die individuellen Schwankungen so gut als möglich auszuschalten und einen Mittelwert zu erhalten, der uns als Maßstab für die Veränderungen allein dienen konnte.

Aus diesem Grunde entstand die erste Reihe von 200 je zu Anfang und am Schlusse der Kur untersuchten Patienten.

Die Zahlen zeigen uns folgendes:

Im ersten Stadium (72) finden wir eine Reaktion, die im großen ganzen derjenigen bei Einwohnern entspricht. Immerhin finden sich auch in dieser Serie eine Anzahl von Fällen, bei denen es nicht zu einer Vermehrung der R. bis zur wahrscheinlichen Norm gekommen ist. Es sind dies 10 weibliche und 4 männliche Patienten, die alle eine sehr gute Kur mit Erreichung der vollen 
Arbeitsfähigkeit hinter sich hatten, und die trotzdem nicht die R-Zahlen des Hochgebirges, zum Teil nicht einmal die normalen Mittelzahlen des Tieflandes erreichen konnten.

Alle diese Fälle zeigen als Kompensation eine ganz erheblich größere $\mathrm{Zu}$ nahme des $\mathrm{Hgb}$, was sich an dem starken Steigen des F I am deutlichsten demonstrieren läßt. Der mittlere Hgb-Gehalt betrug hier bei 10 Frauen 88,2 , bei 4 Männern 94,0, der F I bei Männern 0,98, bei Frauen 1,02, im Mittel 1,00 gegenüber der mittleren Norm unserer Reihe Gesunder von 0,85. Die Vermehrung der $R$ unserer ganzen Reihe I. Stadiums beträgt demgegenüber 0,70 Mill. bei Männern, 0,72 bei Frauen, die mittlere Vermehrung des Hgb derselben Gruppe 9,37 bei Männern, 8,00 bei Frauen, im Durchschnitt für beide Geschlechter 8,67. Während bei genannten 14 Patienten die R-Vermehrung bei den Männern im Mittel $=0$ war, bei den Frauen sich auf nur 0,21 Mill. belief, während die Hgh-Vermehrung bei den Männern durchschnittlich 15,0, bei den Frauen 9,0 ausmachte. Wir sehen also auch hier wieder die beiden Reaktionstypen auftreten, wie wir sie schon bei den Gesunden gesehen haben. Die große Mehrzahl reagiert hier mit einer $R$-Vermehrung, der nach gewisser Zeit eine Hgb-Mehrproduktion folgt, die über das im Tiefland als Maß geltende hinausgeht und eine leichte Vergrößerung des F I mit sich bringt. Eine Minderzahl, die aber doch nicht zu übersehen ist, reagiert anders. Die R.Vermehrung bleibt, in gegenüber dem Mittel sehr bescheidenen Grenzen, während die Hgb-Vermehrung eine exzessive wird. Dadurch steigt der F I auf ganz erhebliche Werte, die weit über dem durchschnittlichen Mittel stehen. Dabei sei betont, daß im Mittel der ganzen Gruppe selbstverständlich auch die 14 Leute der anderen Reaktionsart mitgezählt und im Mittel verwertet sind. In Wirklichkeit weicht also das Mittel noch stärker ab.

Kein einziger der genannten 14 Patienten weist dagegen eine Hgb-Verminderung, keiner eine Herabsetzung des F I auf, so daB sie eine wohl charakterisierte Klasse für sich bilden.

Daß es sich hier nicht um Zufälligkeiten, sondern um biologische Reaktionen handelt, steht für uns fest, schon darum, weil die Resultate verschiedenen Zeiten entstammen und teilweise auch von verschicdenen, aber mit unseren Kammern gut eingeübten Untersuchern ausgeführt wurden. Wir werden zudem diesem Reaktionstypus noch weiterhin begegnen. Daß es tatsächlich ein biologischer Vorgang sein muß, geht auch daraus hervor, daß wir diesen Typus auch beim Gesunden finden. Er hängt also nicht mit der Krankheit zusammen, sondern findet sich auch unter physiologischen Verhältnissen, wie wir überhaupt einen EinfluB einer Lungentuberkulose I. Stadiums auf die Reaktionsfähigkeit des Blutes beim Wechsel vom Tiefland in eine Höhenlage von $1800 \mathrm{~m}$ nicht nachzuweisen imstande sind. Diese Formen reagieren ganz so wie Gesunde. Man darf darum sagen, daß ein Kranker, der mit seinem roten Blutbild wie ein Gesunder reagiert, in dieser Beziehung durchaus normale Reaktionsfähigkeit besitzt und einer Hochgebirgskur von dieser Seite nichts im Wege steht.

Bei den zweiten Stadien macht sich die Krankheit bereits in dem Sinne bemerkbar, daß die R-Endzahlen niedriger stehen als im ersten Stadium. Besonders macht sich dies bei den Frauen geltend. Dabei ist zu berücksichtigen, daß ein Teil dieser Patienten bereits mit einer erheblichen sekundären Anämie 
ankam, also die Knochenmarksfunktion von vornherein gestört war und auch durch die Krankheit weiter ungünstig beeinflußt werden konnte. Die HgbVermehrung ist weniger stark beeinflußt, indem im Durchschnitt immer noch 94,5 bei den Männern, 83,9 bei den Frauen erreicht wurde. Auch die Zunahme ist entsprechend hoch, 10,36 und 7,94. Die Männer zeigen dagegen eine gegenüber dem ersten Stadium geringere R-Zunahme von nur 0,47 Mill, während die bei den Frauen 0,85 Mill. ausmacht. Der Färbeindex beträgt im Mittel der Männer 0,91, der Frauen 0,85.

Das dritte Stadium, unter dem sich sehr viele schwere progrediente Fälle fanden, wie dies beim Material einer Volksheilstätte weiter nicht verwunderlich ist, charakterisiert sich bei den Männern wieder durch ein Ansteigen der R-Zahlen bis 5,30 im Vittel und des Hämoglobins auf 95,8, während sich bei den Frauen ein deutlicher Rückgang auf 4,41 im Mittel und 75,7 beim Hämoglobin ergibt. Dieses Absinken ist durch die Beeinflussung von seiten der klinisch ungünstig liegenden Fälle bedingt. Für die Kranken, die auch klinisch einen Erfolg ihrer Kur erkennen lassen, ergibt sich schon im zweiten Stadium ein erheblich günstigeres Bild, das nur wenig von unserem bei Gesunden gefundenen Mittel abweicht. Noch deutlicher zeigt sich dieser Unterschied bei den Kranken des dritten Stadiums auf negativem Kurerfolg, die im Mittel Minusdifferenzen von 0,59 Mill. bei den Männern und 0,83 bei den Fraven aufweisen.

Auch unter den Fällen des zweiten Stadiums finden wir wieder solche mit abnormem Reaktionstypus, wie oben geschildert. Unter den Fällen dritten Stadiums dagegen nur einen einzigen, der zudem die Verhältnisse quantitativ nur in ganz geringem Maße erkennen läßt. Die Schwere der Krankheit hat hier das Hauptwort, demgegenüber physiologische Unterschiede verschwinden. Die Beurteilung wird darum immer schwieriger, je mohr pathologische Momente das Bild verwischen und es dem Beobachter manchmal unmöglich machen, die einzelnen Faktoren richtig zu werten und in die Gesamtrechnung einzustellen. Bei den schweren Fällen, die mit erheblicher Reduktion der Atmungsoberfläche einhergingen, haben wir nicht mit der Regelmäßigkeit, die Kün dig (3) feststellte, auch ein Ansteigen der $\mathrm{R}$ und des $\mathrm{Hgb}$ finden können. In einigen Fällen mit künstlichem Pneumothorax war dies sehr deutlich, während es anderen mit subjektiver und objektiver Dyspnoe völlig fehlte. Es kommt offenbar auch darauf an, wie rasch sich die Verkleinerung der Atmungsoberfläche einstellt und welche Zeit der Organismus hat, sich an die veränderten Bedingungen anzupassen. Bei einer langsam sich entwickelnden Phthise, die mit Schüben und folgenden Intervallen einhergeht, kann sich sehr wohl auch eine entsprechende Anpassung der Erythropoese einstellen, die dann nicht zu Polyglobulie und stark vermehrtem $\mathrm{Hgb}$-Gehalt führt. Im ganzen fanden wir in der ersten Reihe beim dritten Stadium 7 Fälle mit einer Endzahl von über 6 Mill., was ca. 14\% der Gesamtzahl des Stadiums entspricht.

Keiner unserer Fälle III. Stadiums brachte es auf 7 Mill. Die höchsten Zahlen fanden wir bei zwei Frauen mit schwerem einseitigem Befund mit 6,8 und 6,5. Fünf haben einen gegenüber dem Mittel herabgesetzten F I, nur zwei einen leicht erhöhten, diese zwei auch Hgb-Zahlen von 100 und 110. 6 dieser Patienten haben einen schweren einseitigen Befund mit Kavernen und relativ guter anderer Seite, so daß bei 4 ein therapeutischer Pneumothorax versucht 
werden konnte. Es war aber nur in einem Falle möglich, einen totalen Pneumothorax zu erzielen, die anderen mußten im Verlaufe der Behandlung wieder aufgelassen werden. Es scheint uns darum nicht ausgeschlossen, daß die großen flächenhaften Verwachsungen in diesen Fällen mit zu der relativen Vermehrung der $\mathrm{R}$ beigetragen haben, weil dadurch die ganze schwerer erkrankte Seite praktisch in ihrer Tätigkeit behindert war und darum auch dic sonst noch gut funktionierenden Alveolen, die nach anderen Beobachtungen [Jehn (13)] mitten im tuberkulosebefallenen Gewebe noch erhalten sind und dann meist vergrößert erscheinen, nicht als Kompensation in Erscheinung treten konnten. Gegenüber diesen Kranken mit Polyglobulie fanden sich in diesem Stadium 11 Fälle $=22,6 \%$ mit einer erheblichen Reduktion der R-Zahlen, die auch im Hochgebirge nicht imstande waren, ihren $\mathrm{R}$-Vorrat auf die für das Tiefland geltende Norm zu bringen. Es sind alles infauste Erkrankungen. Es befinden sich darunter zwei Schwestern, die beide im Laufe eines Jahres nach Austritt aus der Behandlung gestorben sind. 8 sind doppelseitig schwer erkrankt. Bei einem war wiederum Pneumothoraxbehandlung nicht möglich. Sie alle zeigen auch tiefe $\mathrm{Hgb}-\mathrm{Zahlen}$ und herabgesetzten $\mathbf{F} \mathbf{I}$.

Die mangelhafte Regeneration von $\mathrm{R}$ und $\mathrm{Hgb}$ ist darum stets als ein signum mali ominis anzusehen und sollte uns veranlassen, solche Kranke so rasch als möglich wieder in das gewohnte Klima zu versetzen, weil sie den Anforderungen, die das Hochgebirge an ihre Reaktionsfähigkeit stellt, nicht mehr gewachsen sind.

Demgegenüber haben 5 der Patienten mit hohen $\mathbf{R}$ Werten einen positiven Erfolg ihrer Kur zu verzeichnen gehabt und befinden sich noch heute nach bis zu 21/2 Jahren wohl. Von einem Patienten haben wir keine Nachrichten mehr, ein letzter endlich ist gegenwärtig mit stationärem Befund wieder in der Heilstätte.

Die hohen R-Zahlen sind darum, wenn sie auch eine erhebliche Reduktion der Atmungsoberfläche nachweisen, prognostisch nicht so schlecht einzuschätzen. Zeigen sie uns doch in hohem Maße die noch erhaltene Reaktionsfähigkeit des Organismus an.

Eine deutliche Knochenmarksinsuffizienz mit Ausschwemmung unreifer Formen ins Blut haben wir mit verschwindenden Ausnahmen von Normoblasten, die ja auch sonst gelegentlich vorkommen können, nie beobachtet. Insbesondere wiesen auch die oben genannten 11 schweren durch das Hochgebirge unbeeinflußbaren Anämien nichts solches auf.

Hatte uns die erste Reihe die Endresultate gezeigt, so interessierte uns nun die Frage: In welcher Zeit reagiert der Organismus unserer Kranken auf die veränderten Bedingungen des Hochgebirges? Ist der Anstieg ein kontinuierlicher oder lassen sich eventuell Schwankungen erkennen?

Wir wählten als Zeiten den 1., den 8. und den 15. Tag, sowie den Tag vor der Entlassung.

Um es vorweg zu nehmen, haben wir auch in dieser Reihe annähernd die?selben Verhältnisse vorgefunden, wie sie von der Reihe der zweimal Untersuchten bereits ausführlich geschildert ist, so daß wir von einer näheren Beschreibung auch der Abweichungen dieser Serie Umgang nehmen können. 
In $42=28,0 \%$ dieser ganzen Reihe konnten wir nach anfänglichem An stieg der $\mathrm{R}$ ein späteres Absinken feststellen, und zwar erfolgte der Hauptanstieg bei 20 Leuten innerhalb der ersten 8 Tage, bei 22 erst innerhalb der zweiten 8 Tage. Bei den übrigen scheint der Anstieg ein kontinuierlicher zu sein, wenn man nur unsere 4 Zahlen berücksichtigt. Daß er es wirklich auch ist, glauben wir nicht behaupten zu dürfen. Die große Zahl derjenigen, die selbst bei unserer lückenhaften Untersuchung entsprechend dem bekannten Weigertschen Gesetze erst einen hohen Anstieg zeigen, dem ein relatives, aber die frühere Zahl nicht erreichendes Absinken folgt, lassen vielmehr den Schluß zu, daB wir noch weit mehr Leute mit demselben Typus gefunden hätten, wenn es die Umstände erlaubt hätten, größere Reihen bei den einzelnen Patienten herzustellen und, wenn möglich, wie dies von anderer Seite schon längst geschehen ist, tägliche Untersuchungen zu machen. Ganz abgesehen davon, daß dies zeitlich bei einer so großen Zahl von Objekten nicht möglich war, konnte eine solche Reihe unseren Patienten, die den wissenschaftlichen Wert solcher Untersuchungen nicht eingesehen hätten, nicht zugemutet werden. Auf alle Fälle sind wir mit den Untersuchungen im Zeitraum von 15 Tagen noch nicht bei allen an der oberen Grenze der Reaktion angelangt, was sich ohne weiteres aus den am Schlusse noch höheren Zahlen ergibt. Die Reaktionszeit ist also für unser Material im Durchschnitt höher als 14 Tage anzuschlagen. In Einzelfällen haben wir denn auch feststellen können, daß mehrere Monate vergehen können, bis sich der Standard herstellt, der der völligen Anpassung entspricht, und zwar gilt dies sowohl für die R- wie für die Hgb-Zahlen.

Die Hauptmasse reagiert zunächst mit einer Vermehrung der $R$, der nach individuell verschiedener. Zeit die Erhöhung auch des Hgb-Gehaltes und damit die Erhöhung des vorher geringeren F I folgt. Das Primäre ist also bei diesen Fällen die Vergrößerung der Oberflä.che in dem oben auseinandergesetzten Verhältnis. Dadurch wird sicher eine vermehrte Ausnützung des vorhandenen, bei der früheren Oberfläche nicht ausreichenden $\mathrm{Hgb}$ gesichert.

In Einzelfällen konnten wir auch in diesem Stadium, d. $\mathbf{h}$. entweder schon am ersten Tage, stärker ausgeprägt jedenfalls am 8 . eine ganz bedeutende Menge von Mikrozyten konstatieren, die schon in der Kammer einen Prozentsatz bis zu $6,5 \%{ }^{1}$ ) ausmachen konnten und auch im gefärbten Präparat entsprechend vertreten waren. In einem besonders interessanten Falle einer Tuberkulose III. Stadiums mit guter Prognose ließen sich sowohl freie Mikrozyten als solche nachweisen, die wie Knospen teils breit aufsitzend, teils gestielt den sonst in Größe und Form durchaus normalen $R$ aufsaßen. Eine direkte Abschnürung konnten wir leider am überlebenden Präparat nie feststellen. In anderen Fällen wieder traten größere, bis $11 \mu$ messende $\mathrm{R}$ auf, die an Zahl ebenfalls erheblich schwankten und keine deutlich dunklere $\mathrm{Hgb}$-Farbe zeigten. Auch sie waren mitunter erheblich vermehrt, doch nie so, daß sie die Hauptmasse der Zellen ausgemacht hätten. Es zeigt dies nur wieder, daß ein und dasselbe Resultat, die Vermehrung der Oberfläche auf zwei verschiedenen Wegen erreicht werden kann, auf dem Wege der Vergrößerung des einzelnen $R$ und auf dem des Austretens kleinster Formen neben den Normalgroßen.

1) In einer Beobachtung. 
Die Verhältnisse bei den sog. chirurgischen Tuberkulosen, die mit 42 Fällen $12 \%$ unseres Gesamtmaterials ausmachen, rechtfertigen eine gesonderte Besprechung.

Im großen ganzen finden wir bei unseren chirurgischen Tuberkulosen geringe Lungenveränderungen, wenigstens was das respiratorische Parenchym anbelangt. Hilusdrüsen dürften wohl bei fast allen festzustellen sein. Dagegen lokalisiert sich hier die Tuberkulose oft gerade an der Bildungsstätte der $R$, und es besteht darum hier eine besonders große Gefahr der ungünstigen Bceinflussung der Erythropoese durch den Sitz der Herde oder auch durch Toxine oder den Erreger selbst. In der Tat stehen unsere chirurgischen Tuberkulosen im Durchschnitt, sowohl was die $\mathrm{R}$ als was die Hgb-Zahl anbelangt, hinter klinisch gleichwertigen Lungentuberkulosen zurück. Dabei sei festgestellt, daß wir bei allen nur 3 therapentische Mißerfolge zu verzeichnen hatten, der klinische Erfolg also trotzdem als ein guter zu bezeichnen ist. Die Therapie bestand außer in den nötigen chirurgischen Eingriffen und orthopädischen Maßnahmen in Sonnenbehandlung, bei schlechtem Wetter ersetzt durch Quarzlampenbestrahlungen des ganzen Körpers. Man hätte deshalb annehmen sollen, daB gerade diese Fälle auf die neben dem klimatischen, vom verminderten Luftdruck herrührenden Faktoren noch der ganz intensive Reiz der Hochgebirgssonne einwirkte, auch ganz besonders gut reagieren würden. Das Gegenteil ist der Fall. Es ist naturgemäß schwer, dem einen oder anderen Faktor den entscheidenden Einfluß zuzuschreiben. Einen Schluß ließe die ebenso systematische Sonnenbehandlung auch von Lungentuberkulosen zu, wozu wir uns aber aus theoretischen Gründen, zu denen wir besonders die Wärmestaunng und die doch nicht von der Hand zu weisende Vergrößerung der Blutungsgefahr rechnen möchten, abgesehen von der ungünstigen Rückwirkung auf das Zentralnervensystem, das uns sogar ab und zu zwang, bei chirurgischen Tuberkulosen trotz der ganz vorsichtigen Steigerung unserer Dosen ein Maximum festzulegen, das unter dem üblich Erträglichen stand, sowie aus den sich daraus ergebenden praktischen Mißerfolgen, die auch wir gehabt haben, nicht entschließen konnten. Die Sonne im Hochgebirge ist sicher nicht so harmlos, wie die heutigen Sonnenfanatiker auch unter den Ärzten glauben möchten, und wenn wir auch die systematische Sonnenbehandlung chirurgischer Tuberkulosen mit ihren besonders funktionell herrlichen Resultaten nie mehr missen möchten, so ist hier doch der Ort, vor dem Zuviel und ganz besonders auch vor der kritiklosen Anwendung dieses Faktors eindringlich zu warnen.

Der Durchschnitt unserer chirurgischen Tuberkulose bleibt mit $\mathbf{R} 5,10$ bei Männern, 4,69 bei Frauen und Hgb 84,5 bei Männern, 76,5 bei Frauen erheblich unter dem Mittel des ersten und zweiten Stadiums der Lungentuberkulose. Ganz besonders fällt bei dieser Gruppe die große Anzahl derjenigen Fälle auf, die nach dem Typus II reagierten. Es sind 12 Patienten dieser Kategorie, was $28,5 \%$ der Gesamtzahl ausmacht. Welche Umstände im Einzelfalle zu diesem Resultate geführt haben, läßt sich nicht bestimmen; die wenigen exakten Untersuchungen über die Einwirkung der Sonnenstrahlung im Hochgebirge haben sich noch nicht mit diesen Verhältnissen beschäftigt. Auch eine $\mathrm{Ab}$ handlung aus letzter Zeit von Rollier und Rossele ${ }^{1}{ }^{\text {) }}$ bringt uns nicht weiter.

1) Rollier und Rosselet, Schweiz. med. Wochenschr. 1921, Nr. 8. 
Möglicherweise bringen uns die Untersuchungen von Dorno ${ }^{1}$ ) in ihrer Auswirkung auf die Therapie der chirurgischen Tuberkulose mit Sonnen- und Quarzlampenbestrahlungen hierüber Aufschluß. Es würde zu weit führen, hier auf diese komplizierten Verhältnisse einzugehen.

Heute stehen sich die Ansichten noch schroff gegenüber. Vgl. dazu Bach ${ }^{2}$ ).

Immerhin ist die Möglichkeit eines besonders starken $R$-Abbaues unter dem Einflusse der Sonnenbehandlung nicht ohne weiteres von der Hand zu weisen (Pigmentation).

In unserem ganzen Material fanden wir 46 Leute mit R-Zahlen über 6 Mill., 5 mit R-Zahlen über 7 Mill. Da die Ursachen, die zu diesen Vermehrungen führten, durchaus nicht immer dieselben sind, möge eine kurze Mitteilung darüber folgen.

Es entfallen auf Leute mit über 6 Mill.:

\begin{tabular}{lrrr} 
& M & F & Total \\
Stad. I & 7 & 8 & 15 \\
Stad. II & 12 & 6 & 18 \\
Stad. III & 8 & 3 & 11 \\
Chir. Tbc. & 1 & 1 & $\mathbf{2}$ \\
\hline Total & $\mathbf{2 8}$ & 18 & $\mathbf{4 6}$
\end{tabular}

Geht man die Krankengeschichten durch, so findet man bei 1 Frau I. Stadiums einen schweren kombinierten Herzfehler, der in der Folge die Rückversetzung ins Tiefland nötig machte. Die übrigen Patienten I. Stadiums zeigen keinerlei Störungen des kleinen Kreislaufes, die eventuell für die hohen Zahlen verantwortlich wären. Sie haben also tatsächlich mit sehr hohen Zahlen reagiert. Im zweiten Stadium finden wir schon 9 Patienten, mit zwei Ausnahmen lauter Männer, bei denen sich eine solche Störung nachweisen läßt. Es handelt sich um zwei exsudative Pleuritiden mit enormem Exsudat und Kompression der einen Lunge, wodurch praktisch die ganze vorher nicht wesentlich erkrankte Seite in kurzer Zeit ausgeschaltet wurde. Die übrigen betreffen 4 doppelseitige Oberlappenprozesse mit teilweise erheblicher Lungenschrumpfung, einer hatte ein Emypem von ca. 31 Inhalt bei doppelseitigen Bronchiektasen; ein letzter endlich litt neben einer Tuberkulose eines Oberlappens an einem schweren Asthma bronchiale mit häufigen Anfällen im Tiefland. Er zeigte zu Anfang den hohen Wert von 6,9 Mill. und ging nach der Kur auf 6,0 hinunter, während sich das Asthma nach einigen kurzen Anfällen völlig gegeben hatte. 6 diese Patienten hatten zudem als Ausdruck ihrer chronischen Zirkulationsstörung typische Trommelschlegelfinger.

Bei den übrigen Patienten zweiten Stadiums gilt dasselbe wie für den Rest des ersten. Auch hier eine Anzahl mit sehr starker Reaktion, die sich zudem bei einem Teil der Fälle erst im Laufe der Zeit geltend machte, was daraus hervorgeht, daß 6 Patienten diese hohe Zahl erst am Ende der Kur erreicht hatten, zwei schon nach 14 Tagen, während wir nach 8 Tagen keine so hohen Zahlen in diesem Stadium feststellen konnten.

1) Dorno, Licht und Luft im Hochgebirge. Braunschweig, Vieweg 1911.

2) Bach, Zur natïrlichen und künstlichen Sonnenbehandlung der Tbc. Dtseh. med. Wochenschr. 1920, Nr. 29. 
Im dritten Stadium steht es ebenso. Bei 7 Kranken ist die Behinderung der Atmung nachgewiesen. Meist sind es schwere einseitige oder dann kavernöse doppelseitige Prozesse. In einem weiteren Falle entwickelten sich die hohen Zahlen nach der Anlage eines Pneumothorax mit vollständigem Kollaps der einen Lunge; bei 3 Kranken ist eine sichere Störung im kleinen Kreislauf nicht festzustellen. Die 8 ersten Kranken entsprechen also derjenigen Kategorie von Kündig (3), bei der auch seinerseits sehr hohe Zahlen gefunden wurden. Auch hier fanden sich bei 8 Kranken die Anzeichen der chronischen Staung als 'Trommelschlegelfinger, bei 5 ferner noch deutliche Zyanose.

Von den sog. chirurgischen Tuberkulosen wurden hohe Zahlen nur bei zwei Leuten gefunden, von denen die eine ebenfalls mit Zirkulationsstörungen in Zusammenhang zu bringen ist. Es handelte sich um einen Mann mit röntgenologisch nachweisbaren, teilweise verkalkten Drüsen im vorderen Mediastinum, wie sich mit Sicherheit aus einer seitlichen Röntgenaufnahme ergab. Es waren schwere Dyspnoeanfälle im Tiefland vorangegangen. Die andere Patientin dagegen ließ Störungen von dieser Seite völlig vermissen.

Zusammenfassend können wir sagen, daß sehr hohe Zahlen, also über 6 Mill., bei unserem Material sowohl durch besondere, uns in den Ursachen noch unbekannte Reaktionsschwankungen des Individuums ihre Ursache haben können, daß sie andererseits namentlich bei schwereren Tuberkuloseformen sicher, durch Störungen im Gebiete des kleinen Kreislaufes bedingt sind und dann als prognostisch ungünstig anzusehen sind, wenn sich auch solche Kranke oft noch überraschend subjektiv wohl befinden und auch arbeitsfähig bleiben können.

Die Leute mit Zahlen über 7 Mill. sind alle leichte Fälle, total 5, 3 Männer und 2 Frauen. Die Zahlen wurden erst am Schlusse der Kur festgestellt, und es fehlen alle Zeichen einer Zirkulationsstörung wie auch einer eventuell in Betracht zu ziehenden Erythrämie. Wir dürfen also hier die erste Ursache die individuell stärkere Reaktion als die gegebene Ursache annehmen.

Die Fälle mit über 6 Mill. machen also 13,1\%, diejenigen von über 7 Mill. $1,1 \%$ unseres gesamten Materials aus. Sie beweisen nur wiederholt, daß es nicht an der Technik, sondern eben am Material liegt, wenn wir im Durchschnitt geringere Zahlen bekamen als die früheren Beobachter.

Beim $\mathrm{Hgb}$ fanden wir ebenfalls geringere Werte, als dies früher der Fall war, immerhin ist dabei in Betracht zu ziehen, daß eine große Zahl der früheren Beobachtungen noch mit der alten Skala rechnete, bei der 100 Teilstriche den heutigen 80 entsprechen. Es sind bei Vergleichen darum stets diese Verhältnisse zu berücksichtigen.

Im allgemeinen haben wir bei unseren Gesunden einen vermehrten Hgb Gehalt und damit einen erhöhten F J finden können. Dasselbe gilt für den Durchschnitt des ersten Stadiums ganz besonders, aber auch bei den anderen Stadien finden wir mindestens verhältnismäßig sehr hohe Hgb-Zahlen.

DaB dafür auch die doch nicht seltenen Leute mit Reaktion vom II. Typus beitragen, versteht sich von selbst.

Die Tabelle am Schluß gibt die Mittelzahlen unseres ganzen Materials nach Stadien und total.

Beiträge zur Klinik der Tuberkulose. Bd. 49. 
Wenn dabei die Frauen unverhältnismäßig niedrige $\mathrm{R}$ - und Hgb-Zahlen aufweisen, so ist dies dem Umstande zuzuschreiben, daß unser Material ein solches einer Volksheilstätte ist, wo die wirtschaftlichen Verhältnisse oft gerade bei den Frauen durch ungenügende Ernährung zahlreiche Geburten und Frauenarbeit auf die Gesundheit in sehr ungünstigem Sinne einwirken und so auch ohne Tuberkulose sekundäre Anämien vorkommen und auffällig oft ein elender Ernährungszustand anzutreffen ist. Die sekundären Anämien sind nicht immer durch das Hochgebirge in dem gewünschten Maße zu beeinflussen und wir waren wiederholt gezwungen, zur Verbesserung dieser Anämien Eisen und Arsenmedikation heranzuziehen. Dicse Fälle sind in der Statistik nicht inbegriffen, weil sonst ein unrichtiges Bild entstünde.

Für unsere spezielle Frage, diejenige der $O$-Aufnahme bei vermindertem O-Druck der Einatmungsluft kommen die Prinzipien einmal der Kolloid-Chemie und dann der Gasdruck in Lösungen in Betracht. Der letztere folgt dem Hen ri schen Gesetze, bis zu einem gewissen Grade auch in kolloidalen Lösungen. Andererseits unterliegt die Aufnahme von $O$ durch das kolloide Hämoglobin den Gesetzen der Adsorption, also wieder einem physikalischen Vorgange. Die Kurven, die aus den Beobachtungen der Resorptionsvorgänge im Blute folgen [Wo. Ostwald bei Bechhold (14)] sind darum keine reinen Adsorptionskurven, sondern sie liegen zwischen diesen und den für den Gasdruck in Lösungen geltenden Geraden des Hen rischen Gesetzes. Wir haben hier also eine Kombination zweier Vorgänge und können uns den Verlauf in concreto folgendermaßen vorstellen:

Der 0 passiert in der Inspirationsphase die Alveolarwand und die Wand der Lungenkapillaren nach den Gesetzen des Gasdruckes. Er gelangt so ins Blutplasma, wo er einen der Menge des $O$ entsprechenden Druck ausübt. Ein Teil des $O$ wird immer noch unter dem Einfluß des Gasdruckes auch zu den $R$ gelangen und dort unter denselben Bedingungen zu den Lipoiden in Beziehung treten. Erst dann erfolgt die Adsorption durch das Hgb. Diese Bindung ist aber keine chemische, sondern eine kolloide, reversible, die in jedem Augenblick auch rückläufig erfolgen kann. Sie ist eine Anreicherung des Absorptums $O$ an der Oberfläche des Adsorbens Hämoglobin.

Die Menge des aufzunehmen möglichen $O$ ist abhängig einmal von der Oberfläche der $R$, an der sich der Vorgang abspielt, weil wir es mit einem hochkolloidalen Körper zu tun haben, für den die Gesetze der Kolloide in Geltung. sind. Es ist bei dieser Vorstellung durchaus denkbar, daß das hydrophile Kolloid Hgb sich an der Oberfläche der $\mathrm{R}$ anreichert und so die $\mathrm{O}$-Adsorption beschleunigt und quantitativ vermehrt wird.

Schon die Gestalt der $\mathbf{R}$ weist auf eine ganz besondere Funktion der Oberfläche hin. Einem relativ geringen Volumen entspricht eine ganz erheblich größere Oberfläche. Wir haben es nicht mit kugelförmigen, sondern mit mehr. weniger scheibenförmigen, nach zwei Dimensionen erheblich stärker entwickelten Gebilden zu tun, während die dritte, die Dicke, stark zurücktritt. Schon diese äußeren Merkmale weisen mit Entscheidenheit auf die Bedeutung der Gesamtoberfläche hin, welche Bedeutung durch den chemischen Aufbau der $R$ weitere Stützen erhält. 
Jedes Kolloid besteht aus mindestens zwei chemisch und physikalisch verschiedenen Komponenten, deren eine an Masse größere das Disperisonsmittel darstellt, während die andere an Masse kleinere die disperse Phase ist. Ein Dispersionsmittel kann aber mehrere voneinander unabhängige disperse Phasen aufnahmen, ebenso kann ein Kolloid erster oder höherer Ordnung, wenn wir uns so ausdrücken dürfen, als disperse Phase in einem gleichfalls kolloiden Dispersionsmittel kolloid gelöst, d. h. suspendiert oder emulgiert sein. Die Selbständigkeit der einzelnen Kolloide ist in dieser Lösung durchaus gewährleistet. Eine chemische Umsetzung braucht nicht stattzufinden und kann sich in sehr mäßigen Grenzen halten, wenn sie vorkommt.

Zum näheren Verständnis dieser Anschauung müssen wir auf einige grundlegende Begriffe der Kolloidchemie eingehen, die wir im wesentlichen nach Bechhold wiedergeben.

Da wir uns die Aufnahme des $\mathrm{O}$ und den Ersatz desselben durch $\mathrm{CO}_{2}$ als einen Asdorptionsvorgang vorstellen müssen, wobei das $\mathrm{Hgb}$ die adsorbierende Phase darstellt, so ist für den Vorgang nicht die Masse, sondern die Oberfläche maßgebend, die bei gleicher Masse sehr verschieden sein kann. Die Adsorption ist bedingt durch die Veränderung der Oberflächenspannung an der Grenzfläche zwischen Lösungsmittel, hier also Plasma und Adsorbens, hier Hgb in Gestalt des $R$.

Die Adsorption ist nach Wo. Ostwald (14) ein mechanischer, kein chemischer Vorgang. Es kommt ihr in vielen Fällen, so auch bei unserem Beispiel die Eigenschaft der Reversibilität $\mathbf{z u}$, indem das adsorptum wieder abgestoßen und durch ein anderes ersetzt werden kann, wie dies in unserem Falle auch tatsächlich in stetem Wechsel erfolgt. Suspensionen und hydrophile Kolloide, zu denen auch unser Hgb zählt, können sich nach den Gesetzen der Oberflächenspannung an der Grenzfläche anreichern, also hier an der Oberfläche der R.

Die kolloidchemische Natur des Hgb wurde erst in letzter Zeit von P. Botazzi (15) untersucht. Nach ihm besteht es aus einem Globulin aus der Gruppe der Histone und der eisenhaltigen Komponente, dem Hämatin, einem Pyrrholderivat. Wir müssen uns das $\mathrm{Hgb}$ als ein in wässeriger Salzlösung suspendiertes Kolloid vorstellen. Daraus erklärt sich sowohl sein elektrochemisches Verhalten als das einer amphoteren Substanz, bei der aber doch die H-IonenDissoziation größer ist als die der OH-Ionen, als seine übrigen chemischen Eigenschaften.

Möglicherweise wirkt das in ca. 3,7\% im $R$ vorhandene Lezithin im Sinne eines Schutzkolloides um die Hgb-Komplexe. Bechhold stellt sich darum die $\mathbf{R}$ als Gebilde vor, die in einem feinen Stützwerk, dem Stroma eingelagerte Tröpfchen, in der Hauptsache Hgb, also eine eiweißhaltige Salzlösung enthalten, welch letztere Tröpfchen von ganz dünnen Lipoidhüllen umgeben sind. Die $\mathbf{R}$ enthalten also ihrerseits drei kolloide Komponenten, Stroma, Hgb und Lipoide (Bech hold, S. 331 und 333). Die Lipoide betragen ca. 3,7\%. Das Hgb316,7\%, Wasser 561\%. In den Beziehungen zwischen $R$ und Plasma gilt folgendes.

Es besteht insofern ein Gleichgewichtszustand zwischen dem Plasma und den $\mathrm{R}$, indem ersteres mehr Na-Salze, letztere mehr K-Salze adsorbieren. Dieser Vorgang ist reversibel. 
Blutkörperchen verhalten sich wie eine hydrophile Suspension (S. 220).

Auch Blut hat eine gewisse Quellungsbreite, wenn sie auch wesentlich geringer ist als die der Organe. Die Quellungsbreite der R beträgt ca. 5\% und ist höher als die Quellungsbreite des Serums mit 2,6\%. Diese Quellungsmöglichkeit wurde von S. G. Hedin (14) nachgewiesen. Sie ist eine Eigenschaft der hydrophilen Gele.

Bechhold (14) bezeichnet darum Bakterien, Hefezellen, Spermatozoen, $\mathbf{R}$ und Leukozyten als hydrophile organische Kolloide ihrer Natur nach und als Ganzes aufgefaßt (S. 306).

Andererseits haben Zsigmondi und Sven berg (16), unabhängig voneinander, die fundamentale Tatsache festgestellt, daß zwischen den molekularen Suspensionen, also den echten Lösungen im chemischen Sinne und den grobdispersen Systemen, den Kolloiden, ein kontinuierlicher Übergang besteht. Sie sind also nicht ihrer Natur nach verschieden, sondern unterscheiden sich nur dadurch, daß im einen Falle die Einzelmoleküle bestehen bleiben, während sie sich im anderen zu mehr weniger großen Komplexen zusammenschließen.

Es steht darum unserem Denken kein Hindernis entgegen, auch hochkolloide Körper, gewissermaßen bereits ko mplexe, organ isier te Kolloide zueinander im Sinne des Dispersionsmittels und der dispersen Phase in Beziehung zu setzen. Wir kommen dann zu der Auffassung, a ch das Gesamtblut als ein solches ko mplexes kolloides Syste m anzusehen, in dem das Plasma das Dispersionsmittel, die $R$ und $W$ dagegen die disperse Phase darstellen. Es erlaubt uns diese Anschauung auch, die Beziehungen zwischen Plasma und $R$ als kolloide Vorgänge zu fassen und entsprechend zu erklären. Diese Ansicht ist durchaus mit der Sahlischen Auffassung des Blutes als Sekret (7) vereinbar.

Wir können uns insbesondere besser vorstellen, warum der $O$ gerade an der Oberfläche der $\mathrm{R}$ seine Wirkung ausübt, weil diese die kolloid-chemisch sowieso wichtige Kontaktfläche der dispersen Phase mit dem Dispersionsmittel darstellt, die auf seiten der dispersen Phase oft besondere Struktur erhält.

Alle Änderungen an der Oberfläche, insbesondere Anreicherung von $\mathrm{Hgb}$ daselbst oder Vergrößerung der Gesamtoberfläche der dispersen Phase müssen einen wesentlichen Einfluß auf die quantitativen Verhältnisse der O-Aufnahme ausüben im Sinne vermehrter Adsorptionsmöglichkeit.

Wir kommen dann zu einem besseren Verständnis unserer beiden Reaktionstypen, indem wir uns den häufigeren Typus I als Oberflächenvergrößerung der dispersen Phase denken müssen. Auch die Ursache für eine solche Oberflächenvergrößerung können wir uns vorstellen, wenn wir uns die Sahlische (7) Auffassung der Ursache der Antikörperanreicherung im Blute zu eigen machen. Die Oberflächenvergrößerung erfolgt hier auch auf einen Reiz hin und dicser Reiz ist die Herabsetzung des O-Druckes im Plasma entsprechend dem in der Höhe herabgesetzten Partialdruck des Luftsauerstoffes.

Wir haben also auch hier als Ursache eine Herabsetzung des Konzentrationsspiegels, verursacht durch Herabsetzung des O-Druckes als Ursache der Mehr. produktion der $\mathrm{R}$ und dadurch der Vergrößerung der verfügbaren Oberfläche anzunehmen.

Bei dem zweiten Reaktionstypus ist wieder die Herabsetzung der O-Konzentration im Plasma dje auslösende Ursache für eine Mehrleistung, aber diese 
Mehrarbeit wird hier nicht durch die Vergrößerung der Gesantoberfläche $\operatorname{der} R$, sondern durch Vermehrung der dispersen Phase des Kolloids Hgb geleistet, was wieder einer Vergrößerung der Oberfläche desjenigen Kolloids gleichkommt, mit dem die 0 -Adsorption erfolgt.

Wir können uns sogar vorstellen, daß diese disperse Phase selbst an der Oberfläche der $\mathbf{R}$ oder in deren nächster Nähe angereichert wird, also an der Kontaktfläche zwischen disperser Phase und Dispersionsmittel, zwischen $\mathbf{R}$ und Plasma, was wiederum ohne Oberflächenvergrößerung aller $\mathrm{R}$ doch einer Oberflächenvergrößerung für die O-Aufnahme gleichkommt.

Die Gesetze der Kolloidchemie, der doch wohl die meisten biologischen Vorgänge folgen, insbesondere diejenigen, die sich im Blute abspielen, auf deren kolloidchemische Natur schon lange $H$. Zangger (6) aufmerksam gemacht hat, bringen uns also das Verständnis gerade desjenigen Vorganges näher, der uns bei der Beurteilung der Wirkungsweise des Hochgebirgsklimas auf den Menschen am meisten interessiert und auch im Effekt am meisten in die Augen fällt, eben die Veränderungen, die das Blut unter dem Einflúß der verminderten O-Spannung in unserer Höhe erreicht. Dabei sei nochmals auf die beim $\mathrm{Hgb}$ studierte Tatsache aufmerksam gemacht, daß, wie schon A. Löwy (17) feststellte, die Aufnahme von $O$ nicht genau parallel dem aufgewendeten Drucke geht, sondern bei stärkeren Konzentrationen im Verhältnis geringer ist als bei schwächeren, was wir uns nicht mit dem Henrischen Gesetz allein, sondern nur mit dessen Anwendung auf kolloidale Lösungen nach den dort herrschenden Gesetzen der mechanischen Oberflächenadsorption erklären können.

Die beiden Typen lassen sich also sehr wohl verstehen, und es ist durchaus im Rahmen der Möglichkeit so hochkolloider Körper, wie sie sowohl im Plasma kreisen, als auch unsere $R$ zusammensetzen, wenn einmal dieser, einmal jener individuell wechselnde Typus der Reaktion zu beobachten ist, weil kleinste Änderungen in den gegenseitigen Beziehungen bereits sehr wesentliche Unterschiede im Endeffekt hervorbringen können.

Wir haben es ja auch nur mit dem Endresultat eines Vorganges zu tun, in dessen Ablauf uns unsere morphologische Technik einzudringen nicht gestattet. Immerhin haben wir auch hicr beim ersten Typus das Gesetz der Uberregeneration mit folgendem Absinken bestätigt gefunden. Auch die abnorm hohen HgbZahlen des zweiten Typus entsprechen durchaus einer solchen Utberregeneration einer Anreicherung unter dem Reiz der Herabsetzung der O-Konzentration des Plasmas, für den wir auch in dem erhöhten Färbeindex unserer gesunden Hochgebirgsbewohner ein Beispiel haben.

Wir haben diese Verhältnisse so eingehend behandelt, weil sie für unsere Aufgabe doch von fundamentaler Bedeutung sind und wir bei dieser Betrachtungsweise einen besseren Einblick in die gegenseitigen Beziehungen zwischen dem $\mathrm{O}$ der Atmungsluft und dem $\mathrm{Hgb}$ bzw. den $\mathrm{R}$ des strömenden Bluts bekommen, wie sie beim Übergang rom Tiefland ins Hochgebirge allmählich eintreten und dort nach gewisser individuell wechselnder Zeit zu bleibenden Veränderungen führen, solange der betreffende Mensch unter dem Einfluß des Hochgebirgsklimas steht. 


\section{R. Hgh und F J im Mittel aller Untersuchten nach Stadien.}

Tabelle I.

350 Patienten beider Reiben.

\begin{tabular}{|c|c|c|c|c|c|c|c|c|}
\hline \multirow{2}{*}{$\begin{array}{l}\text { Stadium } \\
\text { Anzahl }\end{array}$} & \multicolumn{2}{|c|}{ I } & \multicolumn{2}{|c|}{ II } & \multicolumn{2}{|c|}{ III } & \multicolumn{2}{|c|}{$\mathbf{K n}$} \\
\hline & M $(40)$ & W (75) & M (44) & $W(68)$ & M $(36)$ & $W(45)$ & $M(22)$ & $W(20)$ \\
\hline $\mathbf{R}$ & 5,48 & 5,25 & 5,51 & 5,17 & 5,10 & 4,42 & 5,10 & 4,69 \\
\hline $\mathrm{Hgb}$ & 92,6 & 87,7 & 91,0 & 82,6 & 88,7 & 73,8 & 84,5 & 76,5 \\
\hline F.J & 0,85 & 0,85 & 0,82 & 0,79 & 0,87 & 0,83 & 0,83 & 0,81 \\
\hline \multicolumn{9}{|c|}{ Mittel aus allen Stadien inkl. Knochentuberkulose. } \\
\hline & & $\begin{array}{l}\text { nner } \\
\text { iber }\end{array}$ & $\begin{array}{c}\mathrm{R} \\
\mathbf{5 , 3 3} \\
4,92\end{array}$ & & & $\begin{array}{c}\text { FJ } \\
0,84 \\
0,82\end{array}$ & $\begin{array}{c}\text { OQ. } \\
386,9 \\
391,8\end{array}$ & \\
\hline
\end{tabular}

\section{Das weiße Blutbild.}

Viel schwieriger ist das weiße Blutbild zu beurteilen. Einmal ist es auch während der Gesundheit viel größeren Tages- und anderen periodischen Schwankungen unterworfen wie das rote, die Nahrungsaufnahme, die Bewegung, auch psychische Momente, um nur das wichtigste zu nennen, beeinflussen es und lassen als Niederschlag ein von den früheren Untersuchungen an derselben Person abweichendes Resultat zurück.

Unsere Gesunden zeigen folgende Verhältnisse im Mittel:

\begin{tabular}{|c|c|c|c|c|c|c|}
\hline \multirow[b]{2}{*}{ Männer } & \multicolumn{2}{|c|}{ Gesamtzahl } & $\mathrm{N}$ & Ly & Eos & Mono \\
\hline & 7490 & & 4432 & 2240 & 135 & 673 \\
\hline & & in $\%$ & 59,1 & 29,9 & 1,8 & 8,9 \\
\hline Frauen & 8300 & & 5210 & 2408 & 162 & 500 \\
\hline & & in 0 & 62,8 & 29,1 & 2 & 6,0 \\
\hline Mittel beider & 7895 & & 4821 & 2324 & 149 & 586 \\
\hline Normen Nägeli & 7500 & $\begin{array}{l}\text { in } \% \\
\text { in } \%\end{array}$ & $\begin{array}{c}61,8 \\
4500-5000 \\
65-70\end{array}$ & $\begin{array}{c}29,5 \\
1500-2000 \\
20-25\end{array}$ & $\begin{array}{c}2 \\
100,200 \\
2-4\end{array}$ & $\begin{array}{c}7,1 \\
450-600 \\
6-8\end{array}$ \\
\hline
\end{tabular}

Unsere Zahlen zeigen also nur bei den Ly absolut und prozentual höhere Zahlen. Die N sind innerhalb der Norm, die Eos ebenso, während sich die Mono an der oberen Grenze bewegen. Die Gesamtzahl der $W$ ist bei uns jedenfalls nicht vermindert. Große Differenzen bestehen nicht, wenn auch in Einzelfällen hohe Einzelwerte vorkommen, und zwar sowohl hohe Gesamtzahlen, bis 10,000, als auch hohe Einzelquoten, namentlich bei den Ly bis zu $\mathbf{4 4} \%$ bei Männern und Frauen und bei den Mono bis zu $17 \%$ bei Männern, 12\% bei Frauen.

Im ganzen zeigen die Männer den sog. Hochgebirgstypus, als welchen wir nach der Literatur eine prozentuale Vermehrung der Ly und Mono bei absolut verminderter Gesamtleukozytenzahl annehmen, in ausgeprägterer Form als die Fraven.

So große Mono-Zahlen, wie sie Schen $\mathrm{k}$ (10) und $\mathrm{Ruppanner}(18)$ in letzter Zeit gefunden haben, vermißten wir bei unseren Leuten. Es wird sich auch hier in noch höherem Maße als beim roten Blutbild um individuelle Unterschiede handeln, die mit keiner Technik auszugleichen sind.

Unsere Reihen an Kranken lehren uns stets mit dem Bewußtsein einer approximativen und durch viele Nebenumstände beeinflußten Wahrscheinlichkeit folgendes: 
Erste Reihe: Das erste Stadium der Lungentuberkulose läßt keine wesentlichen Abweichungen von unserer Norm erkennen. Die Endzahlen der W stehen mit 7629 der ersten und 7961 der zweiten Reihe sehr nahe am Mittel der gesunden. Die Ausgangszahlen jedoch sind mit 8698 und 7186 erheblich verschieden, so daß man sagen darf, daß die Tendenz besteht, der Norm, wie wir sie festgestellt haben, nahe zu kommen.

Ebenso verhält es sich mit den $N$. Die Annäherung erfolgt auch hier von beiden Seiten nach dem Normalwert auf 4833 und 4916. Dasselbe ist für die Ly mit 2326 und 2242 festzustellen. Die Mono der ersten Reihe erreichen das Mittel nicht, bleiben vielmehr mit 468 darunter, während sie bei der zweiten Reihe mit 634 etwas darüber stehen.

Das konstanteste Element sind unstreitig die Ly. Sie halten sich durch alle Stadien sowohl bei den günstig wie bei den ungünstig verlaufenen Fällen etwa auf der mittleren Höhe. Nur die Knochentuberkulosen machen einen Unterschied, indem dort die Ly sowohl bei den guten wie bei den ungünstigen Fällen höhere Werte $2900=34,0 \%$ und $2800=22,2 \%$ aufweisen. Auch hier erfolgt die Annäherung von beiden Seiten.

Bei allen Formen mit Ausnahme der Lungentuberkulosen ersten Stadiums haben die Gesamtleukozytenzahlen die entschiedene Tendenz zum Steigen. 8800 und 8200 bei Stadium II, 10200 und 12700 bei Stadium III und auch bei den Knochentuberkulosen läßt sich dasselbe feststellen, offenbar wegen des Uberwiegens schwerer, mit Eiterung komplizierter Fälle in unserem Material. Die Zahlen sind hier 8500 und 12600 .

Mit Ausnahme des zweiten Stadiums, wo ein wohl im Material bedingter Minusunterschied bei den Fällen mit negativem klinischen Resultat besteht, ist deutlich eine erhebliche Vermehrung der $W$ bei den ungünstigen Fällen festzustellen.

In erster Linie möchten wir die doch sehr häufige Mischinfektion, die sich im Ansteigen der $\mathrm{N}$-Kurve zu erkennen gibt, verantwortlich machen. Die $\mathbf{N}$ betragen im II. Stadium 5600 und 5900, im dritten 7100 und 9200. Bei den Knochentuberkulosen kommt dies noch deutlicher zum Ausdruck, indem hier die günstigen Fälle rund 5000, die ungünstigen gegen $8000 \mathrm{~N}$ aufweisen, wobei die erste Zahl jeweils dem Mittel aller klinisch-günstigen, die zweite dem der klinisch nicht gebesserten entspricht.

Auffallend ist eine erhebliche Vermehrung der Mono bei den ungebesserten Fällen des dritten Stadiums und bei den Knochentuberkulosen. Es handelt sich bei diesen Vorkommnissen um eine sich in beiden Reihen gleichmäßig wiederholende Erscheinung, die sich bei den mehrfach untersuchten als kontinuierliches Steigen der Mono-Werte von Zahlen im Betrag von 713 und 567 bis zu solchen von 1087 bei Lungentuberkulosen und gar 1200 bei Knochentuberkulosen zu erkennen gibt. Eine Erklärung dafür haben wir nicht, wir können nur die Vermutung aussprechen, daß hier die bei den dritten Stadien, oft ausgesprochene Kachexie mit an der Ausschwemmung dieser Elemente beteiligt ist.

Was den Verlauf der Leukozytenschwankungen während der Beobachtungszeit anbelangt, so läßt sich ersehen, daß in den ersten 14 Tagen eine leichte Schwankung der Gesamtwerte nach unten statthat, der dann der Ausgleich 
in der einen oder anderen Richtung folgt. Bedeutend ist die Schwankung nie gewesen. Einzelfälle, von denen noch die Rede scin soll, abgesehen.

Die Ly sind auch an diesen Schwankungen am wenigsten beteiligt. Am deutlichsten prägt sie sich naturgemä $B$ an den $N$ aus, die ja auch absolut die größten Zahlen aufweisen.

Mono und Eos werden jedenfalls nicht in irgendwie konstantem Sinne beeinflußt, wie es überhaupt außerordentlich schwierig ist, gesetzmäßige Beziehungen in einem so wechselnden Bilde festzuhalten.

Hohe W-Zahlen über 10000 finden wir in allen Stadien, und zwar steigend nach der Schwere der Erkrankung, wie zu erwarten. Es entfallen auf:

$$
\begin{aligned}
& \text { Stadium I . . . . . 35 }=31 \% \\
& \text {,, II . . . . . . } 42=30 \text {, } \\
& \text {, III . . . . . . . } 52=64, \\
& \text { Andere Tuberkulosen . . } 15=35, \text {, }
\end{aligned}
$$

Immerhin haben wir auch im ersten Stadium eine beträchtliche Zahl von Patienten, die mit hohen Zahlen kommen, die wir neben der Mitbeteiligung von bronchialen und peripheren Drüsen, zum Teil auch als Ausdruck noch vorhandener oder eben erst ausgeheilter Pleuritis ansprechen möchten, ganz abgesehen davon, daß wir doch eine beträchtliche Anzahl junger Individuen im 2. Lebensjahrzehnt darunter haben, die in der Anamnese Erkrankungen an Skrofulose aufweisen und bei denen wir einen Habitus lymphaticus noch feststellen konnten. Es betrifft dies letztere 26 Patienten des ersten Stadiums. Bei einer Patientin bestand eine Perikarditis mit concretio Pericardii und schweren Zirkulationsstörungen. Eine weitere hatte noch zur Zeit der letzten Blutuntersuchung eine fieberhafte Bronchitis nicht völlig überstanden, was die hohen Zahlen in der Rekonvaleszenz erklärt.

Doch haben wir auch so noch 15 weibliche Personen, bei denen die Schlußzahlen höher stehen als die Anfangszahlen. Diese haben also trotzdem sie bei uns cine quoad Lungenbefund durchaus gute Kur gemacht haben, keine Anlehnung an das weiße Blutbild des Hochgebirges im Sinne der Verminderung der Gesamtzahl nachweisen lassen. Dagegen fand sich eine stärkere Vermehrung der Ly gegenüber den $\mathrm{N}$ in 7 dieser Fälle, so daB doch in dieser Beziehung die Tendenz zur Ly-Vermehrung nachweisbar ist. Demgegenüber hatten 6 deutliche Vermehrung der N ohne entsprechende Ly-Vermehrung, bei dreien konnte als Ursache eine interkurrente akute Infektionskrankheit. festgestellt werden.

Von den 42 Patienten. II. Stadiums haben 20 eine schlechte Prognose, 3 von ihnen sind im Laufe der Zeit ihrer Krankheit erlegen. Bei 10 stellten wir in der Heilstätte bereits Beteiligung des Larynx fest, einer hatte ein tuberkulöses. Empyem, ein weiterer einen Lupus der Nase, einer eine Lues, ein letzter endlich ein schweres Asthma bronchiale. Die Zahlen der nicht mit anderweitigen tuberkulösen Herden komplizierten Fälle II. Stadiums, bei denen hohe WZahlen gefunden sind, beträgt 29 inkl. 10 mit ungünstigen Heilungsaussichten mit Rücksicht auf den Lungenbefund. Es waren mit Ausnahme von 6 alles offene Tuberkulosen, 2 außerdem kompliziert durch Vitium cordis.

Die 42 Patienten lassen sich ferner noch dahin gruppieren, daß bei 22 die Anfangszahlen niedriger sind als die Schlußzahlen. Die Vermehrung betrifft. 
in 4 Fällen mit klinisch guter Prognose, darunter 2 geschlossene Fälle, in der Hauptsache die Ly, in 3 Fällen sind beide Kategorien gleichmäßig beteiligt, während in 15 Fällen, zu denen auch 8 der erwähnten Larynxerkrankungen zählen, die Vermehrung im wesentlichen auf Konto der $\mathrm{N}$ zu setzen ist. Demgegenüber stehen 20 Patienten, deren W-Zahl im Laufe der Behandlung abnahm, und zwar nahmen in 7 Fällen in der Hauptsache die $\mathrm{N}$ in $6 \mathrm{~N}$ und Ly in 7, dagegen nur die Ly ab. Sieht man sich diese Patienten nach ihrem klinischen Erfolge an, so stellt man fest, daß bei 5 Patienten die Reduktion der Ly-Zahlen im Sinne einer Annäherung an die für unser Material festgestellten Mittel erfolgte, diese 5 Fälle haben alle gute Prognose und mit einer Ausnahme keine Komplikationen, zwei haben schlechte Prognose mit Ly-Abfall weit unter die Norm. Bei den 6 Mann mit gleichmäBigem Abfall halten sich drei mit guter und drei mit schlechter Prognose die Wage. Bei denjenigen mit N-Abnahme fallen wiederum 5 auf gute, nur zwei auf schlechte Prognose. Man kann also sagen, daß eine Reduktion der vorher sehr hohen Ly-Werte auf die annähernde Höhe des Mittels als günstig gewertet werden kann, ebenso ein Abfallen der $\mathbf{N}$ mit Bleiben der Ly, also eine prozentuale Verschiebung zugunsten der Ly, alles selbstverständlich nie als einziger Anhaltspunkt, sondern. stets nur im Rahmen des ganzen klinischen Bildes.

Im dritten Stadium sind die Verhältnisse naturgemäß noch ungüstiger für eine Analyse. Die große Masse dieser Fälle ist schr schwer und die Mischinfektion die Regel.

Bei 18 dieser Patienten ergab sich im Laufe der Behandlung ein relatives Absinken der W-Zahlen, und zwar sanken dic Ly-Zahlen wiedeı um bei 4 Patienten aber erheblich unter die Norm, die Prognose war schlecht, demgegenüber ist sie relativ gut bei 4 Patienten mit Sinken der N-Zahlen. 4 mit guter, 7 mit schlechter Prognose zeigen das Fallen etwa im selben Maße bei beiden Zellarten.

In 34 Fällen stieg die W-Zahl noch während der Behandlung an. Nur ein Fall mit Ansteigen der Ly und drei weitere mit Ansteigen beider Zahlen sind klinisch günstig, alle anderen als ungünstig zu bezeichnen; in je 15 Fällen stiegen beide Zellarten in gleicher Weise oder die $\mathrm{N}$-Zahlen allein.

Uberblicken wir die Reihen, so sehen wir in dem isolierten Steigen der NZahlen und in dem isolierten Zurückgehen der Ly unter die Norm bei gleichbleibenden N-Zahlen, ein ungünstiges Omen im Steigen der Ly, bei zurückgehenden N-Zahlen und im Annähern der Ly-Zahlen an die Norm ein günstiges Moment. Gerade bei leichteren Fällen prägt sich dies besser aus als bei den schweren, wo die Mischinfektion die N-Zahl wohl davernd auf einer erheblichen Höhe zu halten vermag, und Komplikationen, die einen tuberkulösen Herd mit der Außenwelt in Berührung bringen, wie Hauttuberkulosen und Larynxaffektionen wirken im selben Sinne ein.

Charakteristisch ist in diesem Sinne das Bild bei den chirurgischen Tuberkulosen.

Außer einem Falle mit Mediastinaldrüsen sind noch drei Fälle mit ausgedehnter Drüsentuberkulose beteiligt, ferner 7 offene Knochen- und Weichteiltuberkulosen mit sicherer Mischinfektion, ferner zwei mit Peritonitis und Pleuritis exsudativa im Sinne der Polyserositis-Tuberkulose. Nur bei zwei geschlossenen 
Knochentuberkulosen konnte keine direkte Beziehung zwischen der Art der Erkrankung und der Vermehrung der W klinisch sichergestellt werden. Eine deutliche Vermehrung der Ly oder wenigstens eine Verminderung der $\mathbf{N}$ zu. gunsten der Ly ist bei diesen Fällen, wo meist beide Zellformen an der Veränderung beteiligt sind, nicht nachweisbar.

Zweite Reihe: Über die zeitliche Veränderung der W-Zahlen sagen unsere Tabellen folgendes: Die Gesamtzahlen fallen nach 8-14 Tagen etwas unter die Anfangszahlen, um hernach in allen Stadien und bei klinisch günstigen wie ungünstigen Fällen wiederum anzusteigen. Der Anstieg ist ein erheblicher im I. Stadium, ferner bei den klinisch günstigen Fällen des zweiten und den chirurgischen Tuberkulosen, endlich bei den klinisch ungünstigen des dritten Stadiums, während die klinisch ungünstigen des zweiten wie die günstigen des dritten keinen so hohen Anstieg mehr zeigen. In diesen beiden Fällen sind die Schlußzahlen geringer als die Anfangszahlen, in allen anderen Fällen dagegen stehen sie darüber. Immerhin erreichen die Differenzen mit Ausnahme der ungünstigen Fälle des dritten und der chirurgischen Tuberkulose keine 2000 .

Fast genau parallel diesen W-Zahlenschwankengun gehen dic Schwankungen der N-Zahlen. Mit Ausnahme der günstigen Fälle dritten Stadiums, wo wir leichtes Falles feststellen konnten, haben wir überall ein Steigen der N-Zahlen am Schlusse über die Ausgangszahlen. Ebenso entsprechen die zeitlichen Schwankungen der $\mathbf{N}$ bei den $Z$ wischenuntersuchungen in allen Stadien genau den Schwankungen der Gesamtleukozytenzahlen.

Demgegenüber sind die Schwankungen der Ly-Zahlen sowohl wie derjenigen der Mono weder konstant, noch irgendwie charakteristisch zu nennen. Dasselbe gilt für die nur wenig veränderten Eos-Zahlen, die überhaupt die geringsten Schwankungen zeigten, während die Mono-Zahlen durchwegs am Schluss höher stehen als zu Anfang. Auch die Ly-Zahlen bringen es wohl in allen Stadien auf etwas höhere Ziffern, die Differenzen sind aber sehr gering und nicht im Verhältnis der N-Vermehrung.

Unsere W-Werte haben uns also keinerlei sichere Anhaltspunkte für eine prognostische Beurteilung ergeben. Die Gründe liegen sicher in den individuell außerordentlich wechselnden Gesamt- und Teilzahlen bei der Tuberkulose, die von den verschiedensten, uns nur zum Teil bekannten Umständen beeinflußt werden. Auch relativ große Reihen ergaben hier entgegen den Befunden am roten Blutbild keine klaren Differenzen, und darum sind wir auch nicht in der Lage, bindende Schlüsse aus unseren Befunden zu ziehen. Interessant ist immerhin, daß die Annäherung an das weiße Blutbild des Hochgebirges, wie es doch auch aus unseren Reihen Gesunder in abgeschwächtem Maße abzuleiten ist, im W-Blutbild der verschiedenen Tuberkuloseformen im Hochgebirge nicht einmal im ersten Stadium in einwandfreier Art zu finden war. Es ist darum zum mindesten zweifelhaft, ob wir ein weißes Blutbild des Hochgebirges überhaupt annehmen dürfen und ob wir nicht auch hier wieder ein Opfer der kleinen Zahlen geworden sind, die uns wohl zufällige Konstellationen an unserem Material, nicht aber die in Tat und Wahrheit im Mittel vorhandenen Verhältnisse ergeben haben.

Zum allermindesten ist es sehr gefährlich, aus einer relativ kleinen Zahl von Beobachtungen schon Schlüsse für ein eventuell allgemein gültiges Gesetz 
ableiten zu wollen und wir verzichten darum bezüglich des weißen Blutbildes auf eine solche Verallgemeinerung auch unserer Resultate.

\section{Literatur.}

Bei der ausgedehnten Literatur, die wir sowohl über das Blutbild der Tuberkulose als auch über die physiologische Einwirkung des Wechsels aus dem Tiefland ins Hochgebirge haben, konnte es.sich nicht um die Berücksichtigung aller einschlägigen Axbeiten handeln, wir werden darum nur die wichtigsten Arbeiten näher heranziehen und die anderen nur, wenn sie Neues bringen oder mit unseren eigenen Untersuchungen nicht vereinbar sind, bèrücksichtigen.

a) Physiologie der Hochgebirgswirkung auf die $R$ und das Hgb.

Römisch (1) fand bei 30 Leuten in Arosa regelmäBig Vermehrung der $\mathbf{R}$ und des Hgb. Durchschnitt von 21 Männern, ob gesund oder nicht, ist unbestimmt, 6,497 Mill. in Thoma-Kammer, 9 Frauen 5,459 Mill. Bei 4 gesunden Männern fand sich eine durchschnittliche Vermehrung um 0,695 Mill., bei drei leicht anämischen Frauen 0,680 Mill., eine Gesunde fällt außer Betracht.

Egger (1) fand bei 27 gesunden Eingeborenen von Arosa Durchschnittswerte von 7 Mill. Bei 27 aus dem Unterland eingewanderten Personen stets eine Steigerung der R im Mittel von $16,6 \%$ in durchschnittlich 15 Tagen. Auch nach längerer Zeit nahm die Zahl der $R$ noch zu. Letzteres entspricht auch unseren Beobachtungen unter Berücksichtigung des individuellen Faktors.

Experimentell stellten als erste Jaquet und Suter (1) die Vermehrung der $\mathbf{R}$ fest. Das Primäre ist nach ihnen und Egger die vorgängige Vermehrung der $\mathbf{R}$ und später des Hgb. Abderhalden (1) hat in seinen bekannten Versuchen bei Kaninchen eine einmalige primäre, rasch eintretende R-Vermehrung gefunden und vollkommenen Parallelismus mit dem $\mathrm{Hgb}$ festgestellt. Es ist dabei zu erinnern, daß das Experiment beim Tier nicht stets eine direkte Übertragung auf dieselben Verhältnisse beim Menschen erlaubt. Unsere Erfahrungen an großen Reihen sprechen für eine mehr weniger getrennte Beeinflussung, aber stets im selben Sinne der Verbesserung der physiologischen Funktionen.

Die Beeinflussung des Knochenmarks durch die Versetzung in die Höhe im Sinne der Wiederaufnahme der Funktion hat Zuntz (1) bekanntlich an Hunden nachgewiesen.

In neuerer Zeit hat Craandyk (2) auf Grund der Bürkerschen Versuche an 4 Personen die Frage nochmals an Hand zahlreicher Blutabstriche von Gesunden und Kranken in Davos $1500 \mathrm{~m}$ nachgeprüft.

Er kommt wie Bürker (1) zu geringeren Mittelwerten für die $R$, nämlich bei 8 gesunden Männern 5,49 Mill., bei 13 Frauen 5,09 Mill. Hgb bei den Männern, 101, bei den Frauen 90,8 nach Sahli.

Bürker (1 und 2) und Mitarbeiter gelangen auf $1875 \mathrm{~m}$, Schatzalp, bei täglich durchgeführten Untersuchungen auf einen Durchschnitt der 4 Männer von 5,39 Mill. und 96,2 Hgb nach Sahli bei Berücksichtigung aller Werte.

Kündig (3) und van Voornveld (4) berechnen nach Gowers. 
Das Material von Wanner (19) in Villars, $1255 \mathrm{~m}$, bezieht sich auf nur 3 Männer und 2 Frauen. Es beträgt im Mittel für die R 5,43 Mill. bei Männern, bei Frauen 4,83 Mill.

Fießler (20) endlich fand bei Laboratoriumsversuchen im Bürkerschen Institut mittels herabgesetzten Luftdruckes gleichmäßiges Ansteigen der $\mathbf{R}$ und des Hgb. Bei Drucken von 320-430 mm Hg kommt er auf R-Zahlen, die 7 Mill. nicht erreichen, wiewohl die Druckerniedrigung ganz erheblich diejenige in der Höhe von Arosa übersteigt. Sie entspricht einer Höhenlage von 5000-4000 M. ü. M. Er hat übrigens auch sehr hohe Ausgangszahlen, die mit seinem kleinem Material in Beziehung stehen. Seine durchschnittliche Vermehrung beträgt $12 \%$.

Dort auch Literatur bis 1904 über experimentelle Arbeiten.

Die auch unsererseits in Einzelfällen in erheblicher Zahl, in vielen Fällen in kleiner Zahl beobachteten Mikrozyten werden allgemein nach Eggers Vorgang als Formen angesehen, die einer Oberflächenvermehrung gleichkommt, solange das $\mathrm{Hgb}$ noch nicht vermehrt erscheint. Sie wurden auch in unserem Material meist nur bei der ersten und zweiten Untersuchung, sehr selten noch später angetroffen.

Löwy (1) sowie Schaumann und Rosenquist (1) haben die Mikrozyten vermißt. Wir glauben auch hier das Material verantwortlich machen zu müssen.

Die von uns beobachteten Formen entsprechen eigentlich nicht den Mikrozyten sensu strictiori, sondern den Schizozyten. Diese sollen nach Nägeli der Wirkung eines degenerativen Faktors ihr Entstehen verdanken. Dies scheint uns wenigstens für die kleinen Abschnürungsformen, die wir mitunter zahlreich sahen, nicht zuzutreffen, weil dasselbe Blut später diese Formen vollkommen vermissen lieB.

Die Zeit, in der die Regeneration abgelaufen ist, wird von Römisch (1) auf ca. 10-14 Tage angesetzt. Dies trifft für einen Teil der Fälle zu, während andere nach wesentlich längerer Zeit noch Anpassungserscheinungen zeigen können, ohne daß wir einen sichtbaren Grund dafür finden könnten.

Auf dieselbe Zeit kommt auch Egger (1) bei seinen Untersuchungen in Arosa.

Im wesentlichen sind die grundlegenden Versuche Mieschers (1) und seiner Schule, wie auch Zuntz, Müller, Löwy und Casparis (1) sowie Stäubli (21) bestätigt worden, wenn auch besonders unter dem Einfluß Bürkers (1) die anfänglich $\mathrm{zu}$ hoch gefundenen Zahlen für die $\mathrm{R}$ einem tieferen Mittel für Gesunde Platz machen mußten. Unsere Untersuchungen schließen sich an diese Feststellungen an und lassen den individuellen Faktor, der bei einer kleinen Reihe naturgemäß Fehlerquellen schafft, so gut als möglich ausschalten. Sie haben uns zudem noch einen anderen bisher in dieser Form nicht bekannten Reaktionstypus gezeigt.

b) R und Hgb bei Tuberkulose im Hochgebirge.

Als erster hat $\mathrm{K}$ ündig (3) diese Verhältnisse untersucht.

Die Zahlen sind ebenfalls sehr hohe und ganz besonders steigt bei ihm der Durchschnitt seiner Fälle dritten Grades (10 Männer) auf 6,97 Mill., 2 Frauen 
5,73 Mill. Kündig glaubt allerdings seine besonders hohen Zahlen des dritten Stadiums allgemein durch die dann stark reduzierte Atmungsoberfläche erklären zu können. Unsere Zahlen zeigen, daß dies in dieser allgemeinen Form nicht immer zutrifft, daß vielmehr auch Kranke mit ganz erheblicher Ausdehnung ihres Prozesses dann relativ niedrige Zahlen aufweisen, wenn die Krankheit langsam fortschreitet, die Atmungsoberfläche also nur langsam verkleinert und durch cntsprechende Kompensation von seiten emphysematöser Alveolarbezirke auf dem alten Standard gehalten werden kann. Darum zeigen auch bei uns gerade diejenigen Fälle hohe Zahlen, deren Atmungsoberfläche rasch verkleinert wurde.

Andererseits hängt offenbar die starke R-Vermehrung einzelner Fälle mit der erheblichen Reduktion der Atmungsoberfläche ursächlich zusammen. Die Zahlen der von anderen Autoren beobachteten Tuberkulosefälle sind allerdings sehr kleine, bei denen der individuelle Faktor stark mitspielt. Daraus erklären wir uns auch die abweichenden Resultate, besonders von Kündig (3) zu einem schönen Teil.

Außerdem kennen wir noch die Resultate von van Voornveld (4) an 6 Männern und 15 Frauen mit leichten Erkrankungen, 9 Männer und 6 Frauen mit mittelschweren und 2 Männer und I Frau mit schweren Formen von Lungentuberkulose.

Die Zahlen von Craandyk (2) sind, weil ihnen das klinische Bild fehlt, da der Autor sich ein ganz anderes Ziel gesteckt hatte, leider für uns nicht mit zu verwerten. Auffallend ist auf jeden Fall, daß die Zahlen Kündigs durch. weg viel höher stehen als diejenigen von Craandyk.

Ebenso kommt Kündig (3) bei 14 gesunden Männern in Davos auf 6,55 Mill., wobei er keine einzige Zahl unter 6 Mill. hat, was allerdings auffällig ist und im Vergleich mit unseren Resultaten und auch denjenigen anderer, die doch auch einzelne Zahlen untèr 6 und selbst unter 5,5 aufweisen, den Verdacht auf eine technische Beeinflussung des Resultates aufkommen läBt. Bei 10 Frauen fand er 5,80 Mill. Das Hgb ist nach der alten Gowersschen Skala bestimmt.

Als Ablaufzeit der Reaktion bezeichnet er 6 Wochen, kommt aber auch auf längere Zeiten in Einzelfällen.

Die wenigen Bebachtungen Craandyks weisen eher auf ein Absinken der Hgb-Werte bei schwereren Erkrankungen hin.

Craandyk schließt aus seinen Zahlen, daß die Zahlen der $\mathrm{R}$ und des $\mathrm{Hgb}$ bei leichter Erkrankten nicht wesentlich von derjenigen bei Gesunden entfernt seien, was unsere Reihen bestätigt haben.

Unsere Mittelzahlen für Gesunde und Leichtkranke kommen denjenigen von Bürker wohl am nächsten, wenn sie auch technisch ganz anders erreicht wurden.

Es ist dies wohl die beste Stütze für unsere eingangs geäußerte Auffassung, daß nicht die Art der Technik, sondern neben der wirklichen Beherrschung einer der bekannten technischen Methoden, das wesentliche im Material mit seinen von Indidivuum zu Individuum wechselnden Reaktionsmöglichkeiten, die uns zur Aufstellung eines besonderen Reaktionstypus II führten, gelegen ist. 
c) Physiologisches weißes Blutbild.

Erst in jüngster Zeit haben einige Arbeiten von Ruppanner (18) und Schenk (10) versucht, licht in die außerst komplizierten und noch nach mancher Richtung unabgeklärten Verhältnisse der einzelnen Leukozytenarten im Hochgebirge zu bringen.

Schon Römisch (1) fand bei seinen Untersuchten nach erfolgter Akklimatisation Zahlen von 9400 bei Männern, 11100 bei Frauen, dabei sind leider auch Kranke mitgezählt, so daß diese Resultate nicht verwendbar werden; ebenso auch nicht die wechselnden, bald ein Sinken bald ein Steigen aufweisenden Zahlen der Akklimatisationsperiode. Zu jenem Zeitpunkt waren prozentuale Auszählungen noch nicht bekannt.

Wanner (19) fand bei 11 Gesunden auf $1200 \mathrm{M}$ Gesamtzahlen von 7000 bis 8300 , in einem Mittel 5300 , also sehr große Schwankungen, im Mittel 6820. Er stellt eine Vermehrung der Mononukleären gegenüber den Normalzahlen des Tieflandes fest. Dagegen ein Absinken der N-Zahlen. Dasselbe hatte schon Stäubli (21)-St. Moritz gefunden bei 4 Männern und 2 Frauen. Dazu kommt bei Stäubli (21) noch eine Herabsetzung der Gesantleukozytenzahl. Der Prozentsatz der Ly beträgt dort 27,5\%, kommt also unserem Prozentsatz sehr nahe. Wanner (19) bekommt etwas weniger, 24,7\%. Dagegen kamen wir nie auf die hohen Werte von $17,1 \%$ der Mononukleären Stäublis und erreichen auch nicht die $13 \%$ von Wanner. Wanner färbte nach May-Grünwald. Diese Färbung läßt nach Nägeli die Unterscheidung zwischen Mono und manchen Ly nicht zu, was $\mathrm{N}$ ägeli als einen schweren Nachteil der Methode angibt.

Bei Stäubli (21) haben wir die Technik nicht angegeben gefunden, so daß wir dort keine Grüde für die hohen Zahlen finden.

Unsere Zahl in von 7,1\% im Durchschnitt der Gesunden stehen erheblich darunter. Sie sind an gut durchgefärbten Giemsapräparaten erhalten, die die feine Azur-Granulation der Mono besonders gut wiedergibt. Ruppanner und Schenk bekamen folgende Zahlen: Die Gesamtleukozytenzahl ist im Hochgebirge dieselbe wie im Tiefland. Mittelzahl in Samaden 1700 Meter, 6513. Sie stellten fermer ein Absinken der $\mathrm{N}$ und eine Zunahme der Ly fest, für die Schenk ein zur Höhe paralleles Steigen angibt. Die Mono sind leicht vermehrt.

Der Prozentsatz der Ly beträgt dort 33,9. Immerhin hat auch Ruppanner größere Serien von 39 Männern mit $29,2 \%$ Ly gefunden, was unserem Mittel ziemlich nahe kommt.

Schon vor genannten Autoren fanden Baer und Engels mann (22) in Davos Gesamtzahlen bei kleinen Reihen von 5788 im Mittel.

Craandyk (2) ebenfalls bei kleinen Reihen bei Männern 6660, bei Frauen 5930. Auffallende Übereinstimmung herrscht dagegen zwischen unseren Mittelzahlen und denjenigen von Staines und James (23) aus Coloradosprings 2900 Meter, die je 100 junge Leute von 20-30 Jahren untersuchten, sie kommen auf Mittel von 7795 und 7440 , nüchtern in Ruhe. Unser Mittel für Männer ist mit 7490 von dem zweiten Hundert kaum verschieden. 
Bezüglich der $\mathrm{N}$ fanden Baer und Engelsmann (22) $54 \%$ bei wenigen einheimischen Männern durchschnittlich 3159.

Craandyk 53\% mit 3500 bei Männern, 55,2\% mir 3260 bei Frauen. Auffallend ist auch hier. die Übereinstimmung mit $\mathrm{Staines}$ und $\mathrm{James}$, die $55 \%$ und 4287 fanden, was unserem Mittel bei Männern von $59,1 \%$ und 4432 wiederum sehr nahe steht.

Jedenfalls sahen wir auch eine verhältnismäßige Abnahme der $\mathrm{N}$ zugunsten der Ly.

Bei den Lymphozyten finden wir bei Baer und Engelsmann (22) $31 \%$ $=1748$. Craandyk (2) 7 Männer 37,7\% $=2600$, bei 13 Frauen 35,5\% $=2140$.

Die Zahlen von Staines und James (23) sind leider in diesem Punkte unsicher und darum nicht zu' beurteilen.

Die Mono-Zahlen bei Baer und Engels mann (22) $10 \%=587$, mit GiemsaMay-Grünwald, modifiziert nach A Bmann. Bei Staines und James ist leider keine Färbemethòde angegeben, Stäubli (21) ebenfalls, während Wanner (19) aus oben genannten Gründen ebenfalls ausscheidet.

Craandyk $6,4 \%=423$ bei Männern, $6,5 \%=387$ bei Frauen. Färbung May-Grünwald-Giemsa.

Die Eos kommen für unsere Feststellungen nicht in Betracht. Aus dem Vergleich mit der Literatur geht die Gefahr hervor, die eine Beurteilung kleiner Reihen und die Errechnung eines Mittelwertes und einer Prozentzahl aus einer solchen mit sich bringt. Wir bedauern sehr, daB aus äußeren Gründen ein näherer Vergleich der Zahlen von Staines und James (23) mit den unserigen nicht möglich war. In den Fällen, wo dies anging, d. h. bei der Gesamtzahl und derjenigen der $\mathrm{N}$ stimmen unsere Resultate weitgehend zusammen, was nur dahin gedeutetwerden kann, daß nur große Reihen die individuellen Schwankungen derart ausgleichen könnon, daß ein brauchbares Mittel herauskommt.

Wir dürfen also die folgenden Zahlen als Mittel in runden ZahJen für unsere Höhe annehmen:

\begin{tabular}{|c|c|c|c|c|}
\hline $\begin{array}{l}\text { Gesamtzahl } \\
\text { absol, } 7900\end{array}$ & $\begin{array}{c}N \\
4800\end{array}$ & $\begin{array}{c}\mathrm{Ly} \\
2350\end{array}$ & $\begin{array}{l}\text { Eos } \\
150\end{array}$ & $\begin{array}{c}\text { Mono } \\
600\end{array}$ \\
\hline in $\% \quad 100$ & 60 & 30 & 2 & 8 \\
\hline
\end{tabular}

d) WeiBes Blutbild bei Tuberkulose.

War schon die Beurteilung des weißen Blutbildes Gesunder von vielen uns zum Teil noch ganz unbekannten Faktoren, zum Teil noch nicht ganz bekannten und solchen beeinflußt, auf die der Untersuchende nicht einwirken konnte, so kamen bei der Beurteilung einer Tuberkulose noch andere dazu. Einmal übt die Tuberkulose als Infektionskrankheit doch einen gewissen Einfluß aus, dann muß mit der Mischinfektion schon früh gerechnet werden und endlich wird auch das weiße Blutbild wie das rote von der Schwere der Erkrankung, namentlich den toxischen Momenten, die bei der Tuberkulose besonders hoch anzuschlagen sind, naturgemäß beeinflußt.

Dies zeigt sich auch ganz deutlich in der Literatur, die in letzter Zeit bei Gloel (24), zusammen mit eigenen Untersuchungen verwertet ist. Bei fieberlosen Fällen ist durch die Tuberkuloseinfektion allein die Gesamtleukozyten- 
zahl kaum beeinflußt, dagegen Vermehrung bei schwereren Formen, besonders bei exsudativen und kavernösen Prozessen. Höchste Werte in den Endstadien mit starker Destruktion und Mischinfektion, zwischen Ausdehnung der Erkrankung und Leuk-Zahlen bestehen keine Beziehungen.

Gloel fand dagegen bei seinen Morgenuntersuchungen auch in leichten Fällen leichte Erhöhung der Gesundheit mehr als 7000.

Dasselbe konnten wir auch bestätigen. Immerhin sind auch unsere Normalzahlen über 7000 .

Mit steigender Schwere der Erkrankung steigen auch die Leuk-Zahlen, etwa im selben Verhältnis wie in unseren Reihen.

Auch Gloel kann keine prognostische Verwertbarkeit aus seinen Reisen herausfinden:

Während die älteren Autoren keine Abweichungen des Blutbildes bei leichten Tuberkuloseformen gegenüber der Norm fanden, haben neuere doch eine relative Lymphozytose festgestellt. Bei schwereren dagegen aninähernd normales Bild, bei den schwersten endlich deutliche N-Leukozytose. Es entspricht diese letztere Auffassung unseren Resultaten, wobei die vorhandene Ly-Vermehrung durch die in noch stärkerem Maße in Erscheinung tretende $\mathrm{N}$ Ausschwommung gewissermaßen übertönt und damit ausgeschaltet wird.

Dies geht auch aus den Zahlen von Schwermann (25), Steffen (26), Weill (27) und Gloel (24) hervor. Näheres auch bei Nägeli (1) (S. 602ff.).

Von den neueren Autoren hat Galambos (28) in Fällen ersten Stadiums keinen Unterschied gegegnüber Gesunden gefunden. Gloel schließt sich dem für leichte Fälle an. Nach Gloel ist das Verhältnis der $\mathrm{N}$ zu den Ly in Beziehung zu der vorhandenen oder fehlenden Progredienz der Fälle, wie auch wir es für unsere Reihen wenigstens zum Teil feststellen konnten. Die Verschiedenheit des Materials verbietet einen genaueren Vergleich. Bei größeren Reihen würde sich die Zahl derjenigen, die mit relativer Lymphozytose reagieren, auf ein Minimum reduzieren. Nur $\mathrm{N}$ nimmt Gloel einen normalen Prozentsatz von 50-55\% an. Er fand bei einem Prozentsatz von $65 \%$ stets bei genauerer Untersuchung krankhafte Veränderungen. Er bezeichnet darum die Ehrlich'sche Formel als die Krankenhaus-Formel. Ganz unrecht hat er nicht, denn das steht wohl fest, daß jeder Mensch bis in sein drittes Jahrzehnt einmal mit Infektionskrankheiten und dann auch mit anderen eine $\mathrm{N}$. Vermehrung bedingenden Schädigungen Bekanntschaft gemacht hat, so daß eben unsere Normalzahlen in dieser Beziehung auch durch äußere Umstände beeinflußt sind. Stäubli (21) sprach selbst von ciner Staubleukozytose in den Städten, eine Auffassung, die nicht unwidersprochen blieb.

Das Arnethsche Blutbild wird von den Autoren verschieden beurteilt, unsere Auffassung haben wir bereits in dem Abschnitt Technik begründet. Auch Gloel (24) kommt auf anderem Wege zu demselben Resultat.

Bei den 83 Untersuchungen von Baer und Engelsmann (22) zeigt sich auch in schweren Fällen eine N-Leukozytose, während bei den Fällen I. Stadiums eher geringere prozentuale Werte als die Norm gefunden wurden, ca. $65 \%$ gegenüber ca. $55 \%$.

Die Ly schwanken in leichten Fällen um $29 \%$, in schweren um $21 \%$. Die Monozahlen schwanken zwischen 9 und $10,9 \%$. 
Die Gesamtleukozytenzahlen zeigen bei diesen Autoren ein konstantes Verhalten, indem in allen Stadien nach der Kur eine mitunter ganz erhebliche Verminderung der Gesamtzahlen eintrat, Wir können dieses gesetzmäßige Verhalten in unseren weit größeren Reihen nicht bestätigen, wenn wir auch einen gewissen Prozentsatz haben, bei dem dies ebenfalls zutrifft.

Die Zahlen sind nach der tabellarischen Zusammenstellung von Craandyk (2) zitiert.

Die Zahlen von Craandyk sind leider nicht verwertbar, weil seine klinische Einteilung nicht mit der unsrigen übereinstimmt.

Über die an und für sich sehr interessanten Beziehungen zwischen der Leukozytenzahl und der Nahrungsaufnahme, der Bewegung und deren Tagesschwankung bei Tuberkulose geben uns unsere Zahlen keine Anhaltspunkte, weil wir bewußt gerade diese Umstände ausgeschaltet haben, um das Bild nicht allzu sehr zu komplizieren.

Es geht also auch aus der neueren Literatur hervor, daß eine Verwertung des weißen Blutbildes zur Prognose nur unter Berücksichtigung des gesamten klinischen Bildes möglich ist und daß auch so Vorsicht bei der Verwendung am Platze sein dürfte.

Andererseits gehört das morphologische Blutbild zur klinischen Untersuchung, die ohne dieses nicht vollständig genannt werden kann.

\section{E. Zusammenfassung.}

Unsere Resultate führen uns unter Berücksichtigung der Literatur zu folgenden Schlüssen.

1. Nur große Reihèn sind imstande, die individuellen Schwankungen auszugleichen und Mittelwerte für eine bestimmte Höhe zu schaffen.

2. Für das rote Blutbild ergibt sich eine Bestätigung derjenigen Resultate, die wohl eine Vermehrung der $\mathrm{R}$ und des $\mathrm{Hgb}$ fanden, welche Vermehrung aber im Mittel nicht die hohen Werte erreicht, die ihr namentlich von früheren Untersuchern zugesprochen wurde. Bei völliger Beherrschung ist die Technik nicht das Entscheidende. Entscheidend ist vielmehr der in Punkt 1 genannte Faktor.

3. Es konnten zwei voneinander verschiedene Reaktionstypen gefunden werden: Typus I die bekannte Reaktionsform mit primärer Vermehrung der $\mathrm{R}$ und sekundärer Hgb-Zunahme.

Daneben als seltenerer Typus II. Gleichbleiben der $\mathbf{R}$ mit alleiniger Vermehrung des $\mathrm{Hgb}$-Gehaltes.

4. Diese beiden Typen lassen sich nur auf dem Wege erklären, daß die Vorgänge, die zur Vermehrung führen, kolloidaler Natur sind. Die Auffassuing des Gesamtblutes als eines komplexen organisierten Kolloides bringt uns das Verständnis für diese Unterschiede erheblich näher und erlaubt uns einen Einblick in deren Mechanismus.

5. In einem großen Prozentsatz unserer Fälle fanden wir das Weigertsche Gesetz von der pathologischen Regeneration bestätigt. Dieser Prozentsantr ist ein Minimum, bedingt durch unsere Versuchsanordnung.

Beitrăge zur Klinik der Tuberkulose. Bd. 49. 
6. Leichte Formen der Tuberkulose reagieren wie Gesunde auf die Einwirkung des Hochgebirges. Schwerere Fälle lassen eine Beeinflussung der normalen Reaktion durch die Krankheit erkennen.

Diese Abweichungen sind nur dann prognostisch zu verwerten, wenn sie hohe Grade erreichen.

7. Das weiße Blutbild bei Gesunden ergibt im wesentlichen eine Annäherung an das sog." weiße Blutbild des Hochgebirges mit Verminderung der $\mathbf{N}$ und relativer Ly-Vermehrung, die Mono sind in geringem Grade mitvermehrt.

8. Während auch hier leichte Formen von Tuberkulose annähernd wie Gesunde reagieren, macht sich bei Komplikationen und namentlich mit dem Fortschreiten der Erkrankung eine Umkehr im Sinne der N-Vermehrung und Ly-Verminderung geltend, die prognostisch nicht günstig zu beurteilen ist. Immerhin muß jeder Einzelfall auch individuell beurteilt werden und jedes Schematisieren ist hier zu verwerfen.

9. Es gibt kein für die Tuberkulose im Hochgebirge charakteristisches weißes Blutbild, das seinerseits in starkem zeitlichen Wechsel den Gang der Erkrankung im Organismus wiederspiegelt.

\section{Literatur.}

1. Ältere Literatur über Polyglobulie und Leukozyten im Hochgebirge bis 1918 in Nägeli, Blutkrankheiten und Blutdiagnostik. III. Aufl. 1919.

2. Craandyk, M. M., Beitrag zum morphologischen Blutbild im Hochgebirge. Fol hämatol. 23, Heft 2.

3. Kündig, A., Über die Veränderungen des Blutes im Hochgebirge bei Gesunden und Kranken. Korrespbl. f. Schweiz. Ärzte 1897. Heft 1 u. 2.

4. van Voornveld, H. I. A., Das Blut im Hochgebirge. Arch. f. d. ges. Physiol. 92.

5. Schultz; E., Das Blutbild bei endothorakaler Lymphdrüsentuberkulose. Braners Beitr. z. Klin. d. Tuberkul. 21.

6. Zangger, H., Vierteljahrsschr. d. naturforschenden Ges. Zürich 1908.

7. Sahli, H., Über das Wesen und die Entstehung der Antikörper. Schweiz. med. Wochenschrift 1920. Nr. 50 u. 51.

8. Liebmann, E., Zur Methodik der mikroskopischen Untersuchung des Auswurfs. Berl. klin. Wochenschr. 1918. Nr. 41. - Morphologie des Auswurfs bei Lungentuberkulose. Zeitschr. f. Tuberkul. 32, Heft 6.

9. Knoll, W., Zeitschr. f, wissenschaftl. Zool. 95, Heft 1 mit Literatur über Leukozytenmorphologie.

10. Schenk, Th., Über das leukozytäre Blutbild im Hochgebirge. Annalen der Schweiz. Ges. f. Balneologie u. Klimatologie 16.

11. Arneth, J., Die agonale Leukozytose. Münch. med. Wochenschr. Nr. 25, 27, 45 und Monographie. Jena 1904.

12. Brugsch und Schilling, Die Kernform des lebenden neutrophilen Leukozyten beim Menschen (Beobachtungen im Dunkelfeld). Fol. hämatol. 6, 1908. Heft 4.

13. Jehn, W., Die pathologisch-anatomischen Grundlagen der chirurgischen BehandIung der Lungentuberkulose. Bruns Beitr. z. klin. Chirurg. 40, Heft 2.

14. Bechhold, Kolloide in Biologie und Medizin. 2. Aufl. Dresden, Steinkopff 1919.

15. Botaazzi, P., Rend. de. Acad. d. Lincei. 22, 263-270 und Arch. italiano di biologia. 60, 1913.

16. Zsigmondi und Svenberg, Zit. nach Handb. d. ges. Naturwissensch. 2, $1017 \mathrm{ff}$.

17. Loewy, A., in Korany und Richter, Physikalische Chemie und Medizin. Leipzig 1908.

18. Ruppanner, E., Über das leukozytäre Blutbild im Hochgebirge. Schweiz. med. Wochenschr. 1920. Nr. 6. 
19. Wanner, Über das Verhalten der Leukozyten im Höhenklima. Korrespbl. f. Schweiz. Ärzte 1913. Nr. 30.

20. Fießler, A., Wirkung der Verminderung des Luftdruckes auf das Blut. Inaug.-Diss. Tübingen 1904.

21. Stäubli, C., Oberengadiner med. Festschrift 1910. Berlin. L. Simion und 27. Kongr. f. inn. Med. 1910. Wiesbaden.

22. Baer und Engelsmann, Dtsch. Arch. f. klin. Med. 112, 1913.

23. Staines und James, bei Craandyk, S. 27 und Tabellen. Lymphocyte Increase and Altitude. Arch. of int. Med. Sept. 14. Vol. X. S. 376-382.

24. Gloel, W., Beobachtungen über die Leukozyten bei Lungentuberkulose etc. Brauers Beitr. z. klin. Chirurg. 45, 404. 1921. Literatur.

24. Schwermann, L., Blutuntersuchungen bei Lungentuberkulose. Zeitschr. f. Tuberkul. 22, Heft 5.

26. Steffen, L., Utber Blutbefunde bei Lungentuberkulose. Dtsch. Arch. f. klin. Med. 98, 1910. Heft 4.

27. Weill, P., Über Leukozyten und Lungentuberkulose. Zeitschr. f. Tuberkul. 29, Hoft 4 und 30, Heft 1-3. 1919.

28. Galambos, A., Ưber das normale qualitative Blutbild. Fol. hämatol. Arch. 13, Heft 2. 1912. 